\title{
Aeroacoustic Simulation of Nose Landing Gear on Adaptive Unstructured Grids with FUN3D
}

\author{
Veer N. Vatsa*, Mehdi R. Khorrami*, Michael A. Park ${ }^{\dagger}$ and David P. Lockard ${ }^{\dagger}$ \\ NASA Langley Research Center, Hampton, VA 23681
}

\begin{abstract}
Numerical simulations have been performed for a partially-dressed, cavity-closed nose landing gear configuration that was tested in NASA Langley's closed-wall Basic Aerodynamic Research Tunnel (BART) and in the University of Florida's open-jet acoustic facility known as the UFAFF. The unstructured-grid flow solver FUN3D, developed at NASA Langley Research center, is used to compute the unsteady flow field for this configuration. Starting with a coarse grid, a series of successively finer grids were generated using the adaptive gridding methodology available in the FUN3D code. A hybrid Reynolds-averaged Navier-Stokes/large eddy simulation (RANS/LES) turbulence model is used for these computations. Time-averaged and instantaneous solutions obtained on these grids are compared with the measured data. In general, the correlation with the experimental data improves with grid refinement. A similar trend is observed for sound pressure levels obtained by using these CFD solutions as input to a Ffowes Williams-Hawkings noise propagation code to compute the farfield noise levels. In general, the numerical solutions obtained on adapted grids compare well with the hand-tuned enriched fine grid solutions and experimental data. In addition, the grid adaption strategy discussed here simplifies the grid generation process, and results in improved computational efficiency of CFD simulations.
\end{abstract}

\section{Nomenclature}

$\begin{array}{ll}\text { BART } & \text { Basic Aerodynamic Research Tunnel } \\ \text { BDF2OPT } & \text { optimized second-order backward differencing formulation } \\ \text { CFD } & \text { computational fluid dynamics } \\ C_{p} & \left(p-p_{\infty}\right) /\left(0.5 \rho_{\infty} u_{\infty}^{2}\right) \\ \text { DES } & \text { detached eddy simulation } \\ \text { DDES } & \text { delayed detached eddy simulation } \\ \text { FW-H } & \text { Ffowcs Williams-Hawkings } \\ \text { HRLES } & \text { hybrid RANS/LES } \\ \text { LES } & \text { large eddy simulation } \\ \text { MDDES } & \text { modified delayed detached eddy simulation } \\ \text { NLG } & \text { nose landing gear } \\ \text { PDCC } & \text { partially-dressed, cavity-closed } \\ \text { PIV } & \text { particle image velocimetry } \\ \text { psi } & \text { pound per square inch } \\ \text { PSD } & \text { power spectral density } \\ \text { RANS } & \text { Reynolds-averaged Navier-Stokes } \\ \text { SPL } & \text { sound pressure level } \\ \text { TKE } & \text { turbulence kinetic energy, }\left[\left(u^{\prime 2}+v^{\prime 2}+w^{\prime 2}\right) / 2\right] \\ \text { UFAFF } & \text { University of Florida Aeroacoustic Flow Facility } \\ \text { URANS } & \text { unsteady Reynolds-averaged Navier-Stokes } \\ u, v, w & \text { Cartesian fluid velocity components } \\ X, Y, Z & \text { Cartesian coordinates } \\ 2-D \text { TKE } & \text { two-dimensional turbulence kinetic energy, }\left[\left(u^{\prime 2}+v^{2}\right) / 2\right] \\ \theta & \text { circumferential angle, measured clockwise from wheel leading edge } \\ \rho & \text { density }\end{array}$

\footnotetext{
${ }^{*}$ Aerospace Engineer, Computational AeroSciences Branch, Research Directorate; Associate Fellow AIAA

${ }^{\dagger}$ Aerospace Engineer, Computational AeroSciences Branch, Research Directorate; Senior Member AIAA
} 


\section{Superscript:}

perturbation quantity (e.g. $\left.u^{\prime}=u-u_{\infty}\right)$

\section{Subscript:}

$\infty$

free-stream quantity

\section{Introduction}

There has been a growing emphasis on reducing airframe noise to meet the increasingly aggressive standards for permissible noise near airports in recent years. The landing gear is a significant contributor to airframe noise during approach and landing for commercial aircraft, ${ }^{1}$ and therefore, it is important to understand the noise sources associated with such configurations. Prediction of noise sources around the landing gear of an aircraft is an extremely challenging problem despite the progress made in the field of computational fluid dynamics (CFD) during the last several decades. The geometric complexity and highly chaotic unsteady flow fields associated with landing gear pose significant challenges for numerical simulation. In addition to the physical modeling difficulties associated with complex unsteady flows, generating suitable grids that can resolve boundary layer, shear layer and wake regions of interest for such configurations is an extremely demanding and time consuming task.

One possible approach for simplifying the grid generation task is to start with a relatively coarse volume grid that contains well-resolved surface and boundary layer grids adjacent to solid surfaces. Such grids are generally easier and quicker to generate using current unstructured-grid generation codes, such as VGRID. ${ }^{2}$ Once an initial grid is available, adaptive grid techniques can be used to refine the volume grid in high gradient regions of interest.

For the current work, we make use of an unstructured-grid flow code FUN3D, ${ }^{3}$ in which the compressible NavierStokes equations are solved to simulate the viscous unsteady flow field. The FUN3D code has been used for many large-scale applications. ${ }^{3}$ These include simulations of unsteady flow past single- and tandem- cylinder configurations, ${ }^{4}$ where time-averaged and perturbation quantities were shown to be in good agreement with experimental data and earlier computations from the well-established, structured-grid flow code CFL3D. ${ }^{5}$ The FUN3D code has also been used to solve the flow over landing gear configurations and resulting solutions on enriched unstructured grids were shown to compare well with experimental data. ${ }^{6,7}$ However, generation of such hand-tuned enriched grids requires significant human effort, intuition and a priori knowledge of the flow field.

In this paper, we demonstrate the use of a feature-based grid refinement methodology in combination with the outputbased grid adaption technique that has been incorporated in the FUN3D solver. The output-based grid adaption ${ }^{8-10}$ is used here to enrich the volume grid away from the immediate vicinity of solid surfaces to reduce the error in a selected output function, such as drag. On the other hand, the feature-based adaptation focuses on numerical errors of a representative flow feature, such as the vorticity magnitude, and therefore will have the most impact in high vorticity regions. The error estimates on vorticity magnitude are used to construct a metric, ${ }^{10}$ which in turn can be used for generating a target grid density in the computational domain for reducing numerical errors. Once a new enriched grid commensurate with the target grid density is created, the adaption process is repeated until a desired size grid has been generated.

\section{Governing Equations and Flow Solver}

In the present work, the spatially second-order accurate CFD solver FUN3D is used to obtain numerical solutions of the unsteady Navier-Stokes equations. A hybrid approach is used where the Reynolds-averaged Navier-Stokes (RANS) equations supplemented by turbulence models are solved in regions where the grid is inadequate to resolve the unsteady flow features, such as near solid walls, and a large eddy simulation (LES) approach is used in the remainder of the computational domain. The details of the blending between these regions and the subgrid scale model are dependent on the turbulence model used for simulations. ${ }^{11,12}$

FUN3D is a suite of codes, including an unstructured-grid flow solver, adjoint solver, and grid adaption software. The unstructured-grid flow solver developed originally by Anderson and Bonhaus, ${ }^{13}$ has gone through significant modifications over the years by a team of researchers at NASA Langley Research center (LaRC) using modern software practices. ${ }^{14}$ The discrete form of the governing equations is solved either in a time-accurate manner with a constant time-step at every grid point or with variable time stepping to accelerate convergence to a steady state. At each iteration step, a linear system of equations is relaxed with a point implicit procedure. ${ }^{15}$ For the current work, Roe's flux-difference splitting scheme ${ }^{16}$ is used without a flux limiter. 
A dual time-stepping algorithm ${ }^{17}$ with subiterations is employed in FUN3D to converge the solution within each time-step. For these simulations, 15 subiterations per time-step were used, which was adequate to obtain at least 3 orders of magnitude reduction in the residuals of the governing equations. An optimized second-order backward differencing formulation (BDF2OPT) scheme ${ }^{18}$ was chosen for the current work. This scheme produces higher temporal accuracy compared to the standard second-order backward differencing scheme at nominally the same computational cost but with slightly increased memory usage.

The turbulence model under consideration here is based on the one-equation Detached Eddy Simulation (DES) model of Spalart. ${ }^{19}$ Although the DES model has been used often for solving unsteady separated flows, this model depends too strongly on the grid quality and topology, and can lead to non-physical results with grid refinement in viscous layers. Spalart et al. ${ }^{20}$ have subsequently modified the DES model to overcome some of the shortcomings related to its grid dependence resulting in a new version of the model named Delayed Detached Eddy Simulation or DDES. In the DDES model, a blending function that varies between 0 for RANS mode and 1 for LES mode is applied to the destruction terms of the turbulence model. A slight modification of this model was suggested by Vatsa and Lockard ${ }^{4}$ to overcome numerical difficulties associated with the non-physical behavior of the eddy-viscosity observed in the upstream region of cylindrical bluff bodies. In this modified model, the blending function suggested by Spalart et al. ${ }^{20}$ for the DDES model for the destruction terms is also applied to the production terms of the turbulence model, and it will be referred to as the modified DDES, or "MDDES" model, in this paper.

\section{Simulated Configuration and Initial Grid}

The configuration under consideration here is the 1/4-scale, high-fidelity replica of a partially-dressed closed-cavity (PDCC) Gulfstream G550 nose landing gear that was tested by Zawodny et al. ${ }^{21}$ in the University of Florida's Aeroacoustic Flow Facility (UFAFF), an anechoic open-jet tunnel. PIV measurements for the same model were made in the BART tunnel at NASA Langley Research Center. A schematic of the computational domain chosen for current simulations of the nose landing gear configuration in the UFAFF is shown in Fig. 1, where the computational boundaries are displayed. In these simulations, the nose gear assembly is attached to a flat mounting plate, which is suspended in space. The computational domain enclosing the gear assembly and mounting plate is comprised of inflow, outflow, top, bottom and side boundaries as shown in Fig. 1.

Computational results for this configuration obtained on a custom-enriched grid consisting of 145 million nodes were shown by Vatsa et al. ${ }^{7}$ to compare well with measured steady and perturbation pressure data, as well as with farfield noise data. This 145 million node grid is designated as "reference grid" in this paper. As mentioned earlier, it took a significant effort and a priori knowledge of the general flow field to construct this enriched grid. The objective of the current paper is to simplify the grid generation task by using grid adaptation, starting with a rather coarse initial grid. For this purpose, we created an initial grid consisting of approximately 18 million nodes which is designated as "adapt0" in this paper. Planar cuts (shown schematically in Fig. 2) at the wheel midplane and torque arm from this grid are compared with the reference grid in Figs. 3-5. It is clear from these figures that except on the solid surfaces and the adjacent boundary layer regions, the initial grid of 18 million nodes is very coarse and not adequate for resolving the wake regions and component interactions from associated boundary and shear layers. The grid adaptation capability of FUN3D was used to refine this grid in wake and shear layer regions that contain the most energetic unsteady flow created by component interactions and vortex shedding.

\section{Grid Adaption Methodology}

One of the primary objectives of this study was to devise a grid adaption strategy that simplifies the grid generation process for complex configurations, while maintaining the desired resolution in critical regions where elevated flow unsteadiness is suspected. Unstructured grid generation codes, such as the VGRID ${ }^{2}$ are known to be very efficient in generating high density viscous grids in the near-wall regions for complex geometries, as long as the grid quality away from solid surfaces is of secondary importance. We were able to generate an initial coarse grid consisting of approximately 18 million nodes fairly quickly using the VGRID code. Planar cuts from this grid are shown in Figs. 3-5. It is evident from these figures that except near solid surfaces, this grid is very coarse and not suitable for resolving unsteady flow features encountered in the presence of multiple bluff bodies. In addition, the variation of grid spacing from near-wall viscous regions to outer regions is very abrupt and discontinuous (see Fig. 4 (a)), which can lead to large numerical errors during CFD simulations.

A two-step approach for grid adaptation is used here to work with the type of coarse initial grids seen in Figs. 3-5. As a first step, we make use of an output-based grid adaption methodology that requires the solution of steady RANS discrete 
adjoint equations. ${ }^{8-10}$ These linear adjoint equations are solved with a dual-consistent time-marching method developed by Nielsen et al. ${ }^{22}$ A generalized conjugate residual (GCR) scheme ${ }^{23}$ is used with the standard multi-color point-implicit procedure as search directions to improve the iterative convergence. A single-grid error estimation procedure described and demonstrated by Park ${ }^{10}$ for the Euler equations, and subsequently applied to turbulent flow problems by Park et el. ${ }^{24}$ is used here to form an adaptive indicator. A new grid spacing request commensurate with the adaptive indicator and error tolerance is computed using the procedure outlined by Venditti. ${ }^{25}$ Here, the error tolerance is set to half the total remaining error estimate for each cycle of adaptation. The anisotropy of mesh elements is based on the Mach Hessian, where the element size in the smallest spacing direction is dictated by the output-based new grid spacing request. ${ }^{25}$ As shown by Venditti, ${ }^{25}$ numerical errors in the selected output function are reduced by repeated application of this process.

The metric-based grid adaptation scheme for steady problems as described by Park ${ }^{10}$ forms the basis of the grid adaptation scheme used in the next step. This anisotropic adaptation method has also been applied to practical problems without the use of the adjoint by Bibb et al. ${ }^{26}$ Details of the adaptation metric construction that uses vorticity as the adaptive indicator are provided by Shenoy and Smith. ${ }^{27}$ This study uses the vorticity magnitude averaged across the edge connecting adjacent grid nodes (see Eq. (1) of Ref. 27) as the scalar adaptation key along with the Hessian of vorticity magnitude to specify anisotropy and directionality representative of underlying vortical flows. Anisotropy is limited to a maximum and minimum spacing ratio of 6 . A minimum grid spacing constraint of 0.048 grid units was specified to inhibit unbounded growth during the grid adaption process. For viscous grids, the near wall region is frozen and adaptation is only permitted away from solid walls as described by Park and Carlson. ${ }^{28}$ Grid adaptation away from a frozen boundary layer grid can be very useful for problems with a well refined initial boundary layer grid and significant off-body flow features, as demonstrated by Park et al. ${ }^{24}$

The steady anisotropic grid adaptation scheme was extended to unsteady problems using the metric intersection methodology advocated by Alauzet et al. ${ }^{29}$ In this case, a single time window is used that represents a statistically significant time period encompassing the extent of the wake unsteadiness. The time window selected was chosen to capture at least a full cycle of the major peaks and valleys in the unsteady forces generated by the landing gear. Data for averages and spectra are collected after the time-averaged quantities have reached a state of equilibrium on the adapted grids. This strategy eliminates the requirement of time-accuracy-preserving solution interpolation on intermediate grids.

\section{Results}

To match the experimental test conditions, the computations were performed at a freestream Mach number of 0.166 and a Reynolds number of $7.3 \times 10^{4}$ based on the main strut (piston) diameter of 0.75 inches. The computational domain chosen for numerical solutions (see Fig. 1) represents the open jet (no solid boundaries) test set up of the UFAFF tunnel. Since the experiments were conducted with transition strips to ensure turbulent separation, the computations were run in a fully turbulent mode. A constant wall temperature based on flat plate adiabatic wall conditions and no-slip conditions were imposed on viscous surfaces, which included the gear, fuselage and mounting plate. At the inflow plane, total pressure and total temperature corresponding to the wind tunnel conditions were imposed. ${ }^{30}$ Farfield conditions based on Riemann invariants were imposed at the outflow, sides, top, and bottom boundaries.

A close examination of the initial unadapted 18 million node coarse grid that was generated primarily to concentrate grid points in the near wall region indicated very rapid and discontinuous changes in grid spacing between the viscous and inviscid regions as seen in Fig. 4 (a) for the mid-wheel wake region and in Fig. 6 (a) for the region surrounding the landing gear wheel. The flow field associated with such configurations comprising of multiple bluff bodies has two distinct types of flow regimes. The regions upstream of solid surfaces are essentially quasi-steady in nature and encounter low levels of unsteadiness. By contrast, the flow field downstream of the solid surfaces are comprised of highly interactive unsteady shear layers and wake regions. To achieve good quality enriched grids in the entire field, the two-step grid adaption process outlined in the previous section is used here. Specific details of the computations are presented in the following paragraphs. It may not be obvious from Figs. 4-6, but should be mentioned here that the initial 18 million node grid has approximately $50 \%$ fewer surface elements compared to the 145 million node reference grid.

The FUN3D flow solver was run first in a steady mode starting from freestream conditions to expedite the development of mean flow on the initial unadapted 18 million node (adapt0) coarse grid. Using the error in drag as an objective function, the steady-state adjoint approach discussed in the previous section was employed for enhancing the global grid resolution, which resulted in a grid containing 24 million nodes. A planar cut of this grid at the mid-section of the wheel tire is compared in Fig. 6 with the original 18 million node grid. Finer grid density is obtained in the outer part of the viscous region surrounding the wheel for the 24 million node grid compared to the initial unadapted grid. In addition, the grid spacing variation from the near wall region to the outer region in the adapted grid is much smoother all around the wheel including the upstream region. Although not shown here, similar improvement in grid quality is observed near 
other solid surfaces.

The FUN3D flow solver was then run for 30,000 time steps in an unsteady mode on the 24 million node grid before creating the vorticity-based adaptation metric. At free stream velocity, the convective waves can travel nearly 30 times through the computational domain during this period, which is deemed sufficient to purge the initial disturbances out of the computational domain. This was followed by 2000 time-steps, encompassing a representative time interval of the unsteady nature of the flow field to create the metric intersections. This time window was found adequate to capture at least a full cycle of major peaks and valleys in the lift and drag forces generated by the landing gear wheel. By using the feature-based grid adaptation procedure described in the previous section in conjunction with these metric intersections, a new adapted grid was created. After performing a series of refinement cycles, this process resulted in a medium grid with 40 million nodes, and a finer grid consisting of 58 million nodes. These grids are referred to as "adapt1" and "adapt2", indicating the first and second levels of grid adaptation for discussion purposes. Multiple refinement cycles are necessary because the wake is rapidly diffused on initial coarse grids. In order to build up the desired level of grid density, the wake resolution must be grown in a series of adaptive cycles to incrementally increase the vorticity field used to target grid adaptation.

Planar cuts at the wheel midplane and torque arm from the adapted grids are compared in Figs. 7-9. Note that the grid adaptation enriches the grids around the boundary layer edge and in the wake regions, and attempts to capture the downstream development of shear layers and wakes. The reduction in grid-size gradation at the interface of boundary layer and wake regions is evident by comparing grids shown in Figs. 8 and 4 (a). As seen in Fig. 9, the grid adaptation also refined the mesh in the wake region of the strut which is situated upstream of the torque arm, thus capturing the component wake interactions. Increasing the grid size from 40 to 58 million nodes increased the grid density further in the wake and shear layer regions. The grid density for the 58 million nodes in the shear layer and wake regions is comparable to the 145 million node reference grid. On the other hand, the grid density for the 58 million node adapted grid is much coarser in the computational domains where the vorticity is low, resulting in significant savings in grid count and computational resource requirements.

Instantaneous density contours along the torque-arm cut are shown in Fig. 10. Although these results can not be used for direct comparisons due to different time evolution histories, these are still useful for qualitative comparisons. As expected, the wakes and shear layers are resolved much better on finer (adapted) grids. The previous solution from the 145 million node reference grid is also shown in this figure for comparison. It is evident from these figures that the solution on the initial 18 million node grid lacks the resolution in the wakes created by the upstream strut and the torque-arm. The resolution in the wake and shear layer regions improves considerably on the 40 and 58 million node grids. Note that the solution on the 58 million node adapted grid is qualitatively similar to the 145 million node reference grid solution.

Simulations on these grids have been performed for a minimum of 60,000 time-steps, which was found sufficient to produce well-converged time-averaged solutions. The computed time-averaged surface pressure distributions on the port wheel are plotted in Fig. 11 as a function of the circumferential angle $\theta$, which is measured in the clockwise direction from the wheel leading edge. Solutions based on the 145 million node grid from Ref. 7, and the experimental data are also shown in this figure. The agreement of computed pressures with the experimental data improves with grid refinement, the biggest gain seen by going from 18 to 40 million nodes. Except for a slight under-prediction of the expansion peak near the $130 \mathrm{deg}$. circumferential location on the wheel, the agreement between solutions on the adapted grids and the 145 million node grid is quite good.

Experimental measurements were taken along nine rows of ports mounted on the gear door surface as depicted in Fig. 12. The computed pressures are compared with the measured data in Fig. 13 along rows 5 and 7. Because of the sparseness of the experimental data, it is difficult to ascertain the precise shape of the experimental curves and to draw firm conclusions about these comparisons. In general, the computed pressures compare reasonably well with the measurements in the middle portion of the door. There is a steep drop in computed pressure levels towards the door edges because of sudden acceleration of the flow at the door edges which is not captured in the experimental data due to the lack of measurement ports in these regions.

The computed results from the current simulations for the two-dimensional turbulence kinetic energy (2-D TKE), $\left[\left(u^{\prime 2}+v^{\prime 2}\right) / 2\right]$, on the initial unadapted 18 million node grid and the adapted 58 million node grid for a plane downstream of the mid-section of the wheels, denoted as wheel_plane3 in Fig. 14, are compared in Fig. 15 with the 145 million node grid solutions and the PIV data obtained from the experiments conducted in the BART ${ }^{31}$ facility. No such data is available from the UFAFF tests at this time. It is evident that the 2-D TKE levels from the 18 million node grid are underpredicted due to poor resolution of shear layers and wake region downstream of the wheels. For the 58 million node grid, the computed results are qualitatively similar to the measured data and the 145 million node results from Ref. 7, and display the correct shape and trends for the decay of TKE levels in the wheel wake and shear layer regions.

Next, we examine the surface pressure power spectral density (PSD) distributions at different locations on the gear 
corresponding to the position of Kulite ${ }^{\circledR}$ pressure sensors, which are depicted in Fig. 16 for reference. It is important to obtain accurate solutions for surface PSD distributions because these are used as input to noise propagation codes for predicting the farfield noise. The computed PSD distributions from this study are compared with the experimental data in Fig. 17 at two locations on the door corresponding to Kulite ${ }^{\circledR}$ pressure sensors (channels) 3 and 10, and in Fig. 18 at sensors 7 (wheel) and 15 (lower arm). The computational results from the 145 million node grid of Ref. 7 are also plotted in these figures for reference. Even though the surface grids are identical for the 18, 40 and 58 million node grids, a gradual improvement in agreement with the experimental data is observed with adaptive grid refinement. The computations from the initial 18 million node grid, which is very coarse in wake regions, produces the least accurate solutions as expected. The agreement between the 58 million node grid solutions and experimental data is comparable to the reference solutions on the highly enriched 145 million node grid up to a frequency of $3 \mathrm{KHz}$. Note that PSD levels beyond this frequency are dropping rapidly and therefore will have only small effect on the overall sound pressure level of the farfield noise.

\section{A. Farfield Noise}

Zawodny et al. ${ }^{21}$ have conducted aeroacoustic testing in the UFAFF, an open jet anechoic tunnel of the PDCC Gulfstream G550 nose landing gear configuration that is under consideration in this paper. In this experiment, a series of 11 microphones were placed at a distance of several wheel diameters underneath the nose gear centerline along the flyover direction as shown in Fig. 19. The measured spectra at the microphone locations were corrected to account for shear layer refraction. Note that the effective position of microphone 7 shown in Fig. 19 is directly underneath the nose gear, which closely represents an overhead flyover position of $90^{\circ}$.

We have computed the farfield noise with the conventional hybrid CFD/Acoustic Analogy Approach, where the pressure fluctuations on the solid surfaces of the landing gear configuration obtained from the CFD solutions were used as input to the Ffowcs Williams-Hawkings ${ }^{32}$ (FW-H) solver PSU-WOPWOP ${ }^{33,34}$ for computing the $1 / 3$ Octave sound pressure levels (SPL) in $\mathrm{dB}$ at farfield points. The results obtained in this manner at flyover microphones 4, 7, and 9 from the 18, 40 and 58 million node grid simulations are shown in Figs. 20 (a)-(c), respectively. In addition, the results on the reference grid of 145 million nodes from the earlier work of Vatsa et al. ${ }^{7}$ are also presented here for comparison. These computations include the contribution of all the pertinent solid surfaces, i.e. the gear, fuselage and mounting plate. The experimental data from the UFAFF, which represents the noise levels at these locations from all the sources for this configuration, is shown as solid black lines connecting filled circles. Whereas the results based on the initial 18 million node grid show the largest deviation from the measured data, the agreement of computed results in general improves with grid refinement. The results from the 58 million adapted grid case are in reasonably good agreement with the 145 million node results upto a frequency of $2 \mathrm{KHz}$, after which the predicted noise level displays a sharp decrease. At this point, it is not clear whether this behavior is a result of coarser surface grid density used for the adapted grids, or if it is due to lack of resolution in the outer part of the upstream boundary layer regions.

\section{B. Computational Efficiency of Grid Adaption}

So far, we have not discussed the cost incurred by the adaption process described in this paper. In order to simplify the discussion, we will normalize the computational costs for grid adaption by the overall computational time used for the 58 million node case. The computational cost incurred in the first step of grid adaption that used the steady adjoint for grid adaption to create a 24 million node grid was found to be minimal. Multiple feature-based grid adaption cycles were then performed starting with 30,000 unsteady time cycles on the 24 million node grid. A representative time window of 2000 unsteady cycles on each subsequent grid was used for constructing the metric intersections. Most of the computational resources here were consumed by the unsteady flow calculations. Combining all the computational costs required to generate the final adapted grid was approximately $20 \%$ of the total cost to generate the far-field noise data for the 58 million node grid case. Currently there is additional human time involved in this process, but most of these steps can be easily scripted so that the whole process can be performed in an autonomous mode.

It is worth pointing out here that it is not fair to judge the numerical efficiency of the current adapted grids on the basis of purely the grid count from the 145 million node grid of Ref. 7. One of the objectives of that previous work was to test the feasibility of computing the farfield noise directly from unsteady solutions without the need of noise propagation codes. Therefore, this 145 million node grid was designed to provide adequate resolution to capture acoustic signals of up to $10 \mathrm{KHz}$ frequency in the vicinity of microphones used for measuring the farfield noise. This requirement was added to the original criteria of resolving viscous and sheer layers and the wake regions associated with this configuration. A planar cut of this grid is shown here in Fig. 21, where the location of sampling points and microphones are identified. A significant number of grid points are clustered in the dark, high grid density zone, which in 
reality is a cone frustum in 3-D space. In our estimate, approximately 40 million nodes were placed in this conical region underneath the gear to accommodate direct measurement of acoustic signals. If we were to design a comparable mesh for computing farfield noise with the CFD/Acoustic Analogy approach for this configuration, the equivalent grid count would have been approximately 105 million nodes. Even with this caveat, the proposed grid adaption process offers significant savings in computational and grid generation effort for obtaining accurate numerical solutions on smaller meshes, without requiring a priori knowledge of the flow field.

\section{Concluding Remarks}

A grid adaptation methodology in the unsteady Navier-Stokes flow solver FUN3D is used to improve the overall efficiency of obtaining accurate numerical solutions over a complex nose landing gear configuration. The results presented here demonstrate the feasibility of starting with a relatively coarse volumetric mesh, and enriching it in shear layer and wake regions associated with complex configurations to improve the solution accuracy without the need for uniform global refinement. Such methodology has the potential of simplifying the grid generation task for complex configurations in a semi-automatic manner for improved efficiency.

A series of adapted grids were created starting from a coarse grid containing 18 million nodes. Solutions are presented on the coarse grid along with the solutions on the 40 and 58 million node adapted grids. These are compared with previously published solutions on the 145 million node grids and the available experimental data. As expected, the overall solution accuracy improves with grid refinement. The resulting solutions on adapted grids for time-averaged and unsteady quantities are shown to compare well with the experimental data and previous solutions from highly-enriched grids for a nose landing gear configuration.

Computed power spectral densities at selected sensor locations and the farfield noise are also compared with previous solutions and experimental data. In general, the solution accuracy improves as the grid is refined, and the accuracy of numerical solutions on the 58 million node grid are found to be comparable to the reference 145 million node grid solutions for practical purposes.

\section{Acknowledgments}

This work was supported by NASA's Fundamental Aerodynamics and Integrated Systems Research Programs through the Fixed-Wing and Environmentally Responsible Aviation projects. The authors would also like to acknowledge Mr. Michael Wiese of Vigyan, Inc. for generating the initial grids used in this work.

\section{References}

\footnotetext{
${ }^{1}$ Khorrami, M. R., Lockard, D. P., Humphreys, Jr., W. M., Choudhari, M. M., and Van de Ven, T., "Preliminary Analysis of Acoustic Measurements from the NASA-Gulfstream Airframe Noise Flight Test," AIAA Paper 2008-2814, May 2008.

${ }^{2}$ Pirzadeh, S., "Three-dimensional Unstructured Viscous Grids by the Advancing Layer Method," AIAA Journal, Vol. 33, No. 1, 1996, pp. $43-49$.

${ }^{3}$ FUN3D Web page: http://fun3d.larc.nasa.gov, March 2013.

${ }^{4}$ Vatsa, V. and Lockard, D., "Assessment of Hybrid RANS/LES Turbulence Models for Aeroacoustics Applications," AIAA Paper 2010-4001, June 2010.

${ }^{5}$ Rumsey, C., Biedron, R., and Thomas, J., “CFL3D: Its History and Some Recent Applications,” NASA TM 112861, May 1997, presented at the Godonov's Method for Gas Dynamics Symposium, Ann Arbor, MI.

${ }^{6}$ Vatsa, V., Lockard, D., and Khorrami, M., "Application of FUN3D Solver for Aeroacoustics Simulation of a Nose Landing Gear Configuration," AIAA Paper 2011-2820, June 2011.

${ }^{7}$ Vatsa, V., Lockard, D., Khorrami, M., and Carlson, J.-R., "Aeroacoustic Simulation of a Nose Landing Gear in an Open Jet Facility using FUN3D," AIAA Paper 2012-2280, June 2012.

${ }^{8}$ Nielsen, E. J. and Anderson, W. K., "Recent Improvements in Aerodynamic Design Optimization on Unstructured Meshes," AIAA Journal, Vol. 40, No. 6, 2002, pp. 1155-1163.

${ }^{9}$ Nielsen, E. J., Aerodynamic Design Sensitivities on an Unstructured Mesh Using the Navier-Stokes Equations and a Discrete Adjoint Formulation, Ph.D. thesis, Virginia Polytechnic Institute and State University, 1998.

${ }^{10}$ Park, M. A., Anisotropic Output-Based Adaptation with Tetrahedral Cut Cells for Compressible Flows, Ph.D. thesis, Massachusetts Institute of Technology, Sept. 2008.

${ }^{11}$ Lynch, C. and Smith, M. J., "Hybrid RANS-LES Turbulence Models on Unstructured Grids," AIAA Paper 2008-3854, June 2008.

${ }^{12}$ Baurle, R. and Edwards, J., "Hybrid Reynolds-Averaged/Large-Eddy Simulations of a Coaxial Supersonic Free-Jet Experiment," AIAA Paper 2009-0129, January 2009.

${ }^{13}$ Anderson, W. K. and Bonhaus, D. L., "An Implicit Upwind Algorithm for Computing Turbulent Flows on Unstructured Grids," Computers and Fluids, Vol. 23, No. 1, 1994, pp. 1-21.

${ }^{14}$ Alexandrov, N., Atkins, H. L., Bibb, K. L., Biedron, R. T., Gnoffo, P. A., Hammond, D. P., Jones, W. T., Kleb, W. L., Lee-Rausch, E. M.,
} 
Nielsen, E. J., Park, M. A., Raman, V. V., Roberts, T. W., Thomas, J. L., Vatsa, V. N., Viken, S. A., White, J. A., and Wood, W. A., “Team Software Development for Aerothermodynamic and Aerodynamic Analysis and Design," NASA TM 2003-212421, Nov. 2003.

${ }^{15}$ Nielsen, E., Lu, J., Park, M., and Darmofal, D., “An Implicit, Exact Dual Adjoint Solution Method for Turbulent Flows on Unstructured Grids," Computers and Fluids, Vol. 33, No. 9, 2003, pp. 1131-1155.

${ }^{16}$ Roe, P. L., “Approximate Riemann Solvers, Parameter Vectors, and Difference Schemes,” Journal of Computational Physics, Vol. 43, No. 2, 1981, pp. 357-372.

${ }^{17}$ Biedron, R., Vatsa, V., and Atkins, H., "Simulation of Unsteady Flows using an Unstructured Navier-Stokes Solver for Moving and Stationary Grids," AIAA Paper 2005-5039, June 2005.

${ }^{18}$ Vatsa, V., Carpenter, M., and Lockard, D., "Re-evaluation of an Optimized Second Order Backward Difference (BDF2OPT) Scheme for Unsteady Flow Applications," AIAA Paper 2010-0122, January 2010.

${ }^{19}$ Spalart, P. R., "Young Person's Guide to Detached-Eddy Simulation Grids," NASA CR-211032, July 2001.

${ }^{20}$ Spalart, P. R., Deck, S., Shur, M. L., Squires, K. D., Strelets, M. K., and Travin, A., “A New Version of Detached-Eddy Simulation, Resistant to Ambiguous Grid Densities," Theoretical and Computational Fluid Dynamics, Vol. 20, No. 3, 2006, pp. 181-195.

${ }^{21}$ Zawodny, N., Liu, F., Yardibi, T., Cattafesta, L., Khorrami, M., Neuhart, D., and Van de Ven, T., "A Comparative Study of a 1/4-scale Gulfstream G550 Aircraft Nose Gear Model," AIAA Paper 2009-3153, May 2009.

${ }^{22}$ Nielsen, E. J., Lu, J., Park, M. A., and Darmofal, D. L., “An Implicit, Exact Dual Adjoint Solution Method for Turbulent Flows on Unstructured Grids," Computers and Fluids, Vol. 33, No. 9, 2004, pp. 1131-1155.

${ }^{23}$ Eisenstat, S. C., Elman, H. C., and Schultz, M. H., "Variational Iterative Methods for Nonsymmetric Systems of Linear Equations," SIAM Journal on Numerical Analysis, Vol. 2, April 1983, pp. 345-357.

${ }^{24}$ Park, M. A., Lee-Rausch, E. M., and Rumsey, C. L., "FUN3D and CFL3D Computations for the First High Lift Prediction Workshop," AIAA Paper 2011-0936, Jan. 2011.

${ }^{25}$ Venditti, D. A., Grid Adaptation for Functional Outputs of Compressible Flow Simulations, Ph.D. thesis, Massachusetts Institute of Technology, 2002.

${ }^{26}$ Bibb, K. L., Gnoffo, P. A., Park, M. A., and Jones, W. T., "Parallel, Gradient-Based Anisotropic Mesh Adaptation for Re-entry Vehicle Configurations," AIAA Paper 2006-3579, June 2006.

${ }^{27}$ Shenoy, R. and Smith, M. J., "Unstructured Overset Grid Adaptation for Rotorcraft Aerodynamic Interactions," American Helicopter Society 67th Annual Forum, Virginia Beach, VA, May 2011.

${ }^{28}$ Park, M. A. and Carlson, J.-R., “Turbulent Output-Based Anisotropic Adaptation,” AIAA Paper 2010-0168, Jan. 2010.

${ }^{29}$ Alauzet, F. and Olivier, G., "Extension of Metric-Based Anisotropic Mesh Adaptation to Time-Dependent Problems Involving Moving Geometries," AIAA Paper 2011-0896, Jan. 2011.

${ }^{30}$ Carlson, J.-R., "Inflow/Outflow Boundary Conditions with Application to FUN3D,” NASA TM 2011-217181, NASA Langley Research Center, Oct. 2011

${ }^{31}$ Neuhart, D., Khorrami, M., and Choudhari, M., “Aerodynamics of a Gulfstream G550 Nose Landing Gear Model,” AIAA Paper 2009-3152, May 2009

${ }^{32}$ Ffowcs Williams, J. E. and Hawkings, D. L., "Sound Generated by Turbulence and Surfaces in Arbitrary Motion," Philosophical Transactions of the Royal Society, Vol. A264, No. 1151, 1969, pp. 321-342.

${ }^{33}$ Brentner, K. S., Lopes, L. V., Chen, H. N., and Horn, J. F., "Near Real-Time Simulation of Rotorcraft Acoustics and Flight Dynamics," 59th Annual Forum, AHS International, Alexandria, VA, 2003.

${ }^{34}$ Bres, G. A., Brentner, K. S., Perez, G., and Jones, H. E., "Maneuvering Rotorcraft Noise Prediction," Journal of Sound and Vibration, Vol. 275, No. 3-5, 2004, pp. 719-738. 


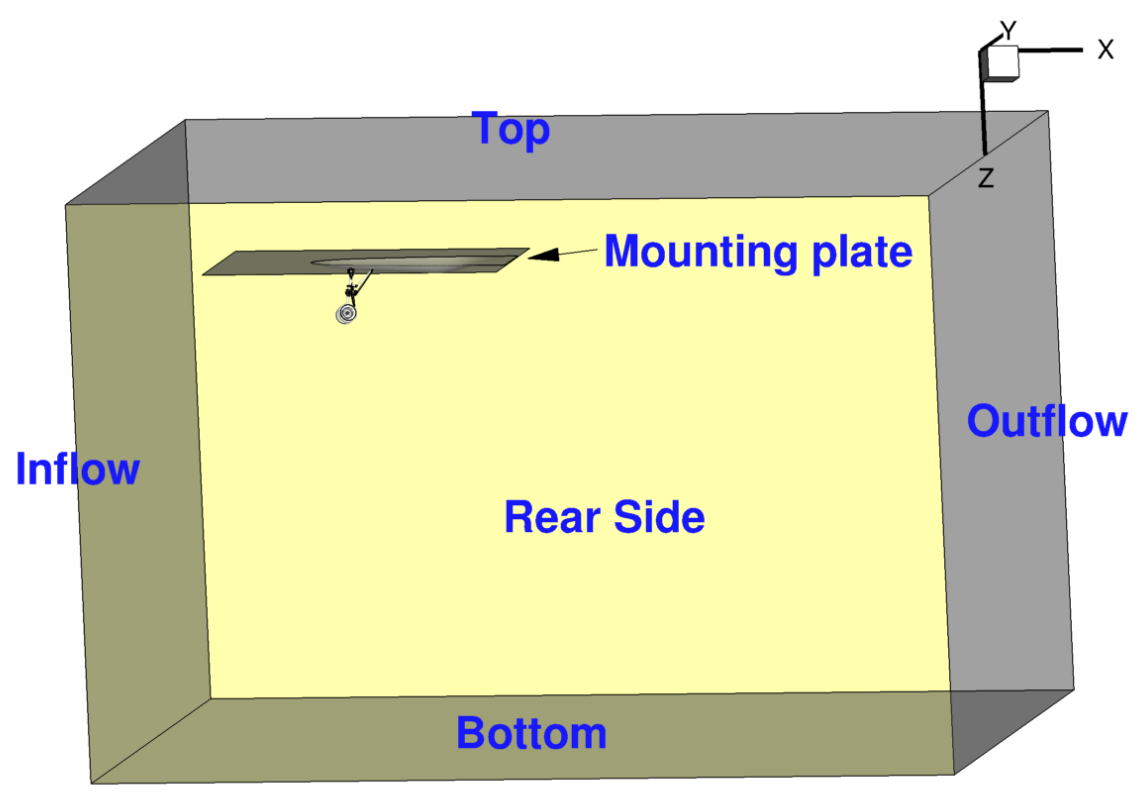

Figure 1. Computational model of the nose landing gear in UFAFF tunnel.

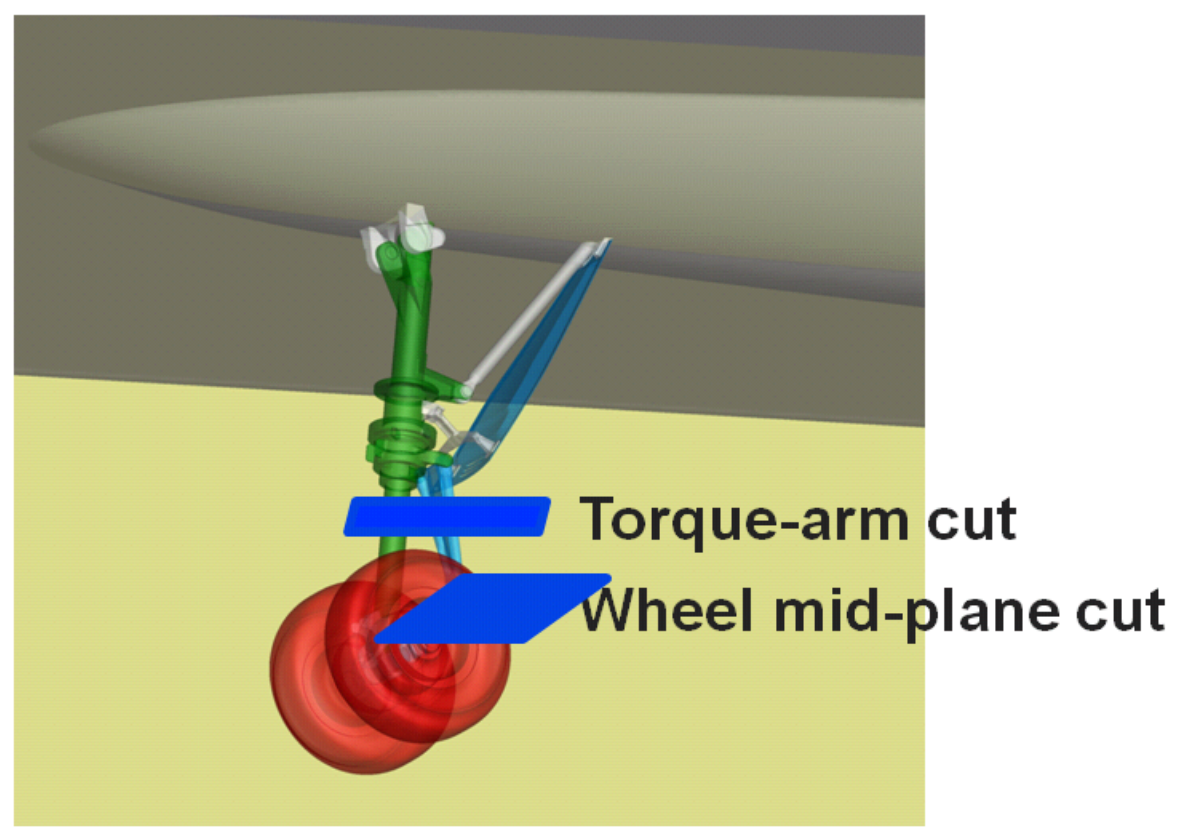

Figure 2. Wheel and torque-arm cut locations. 


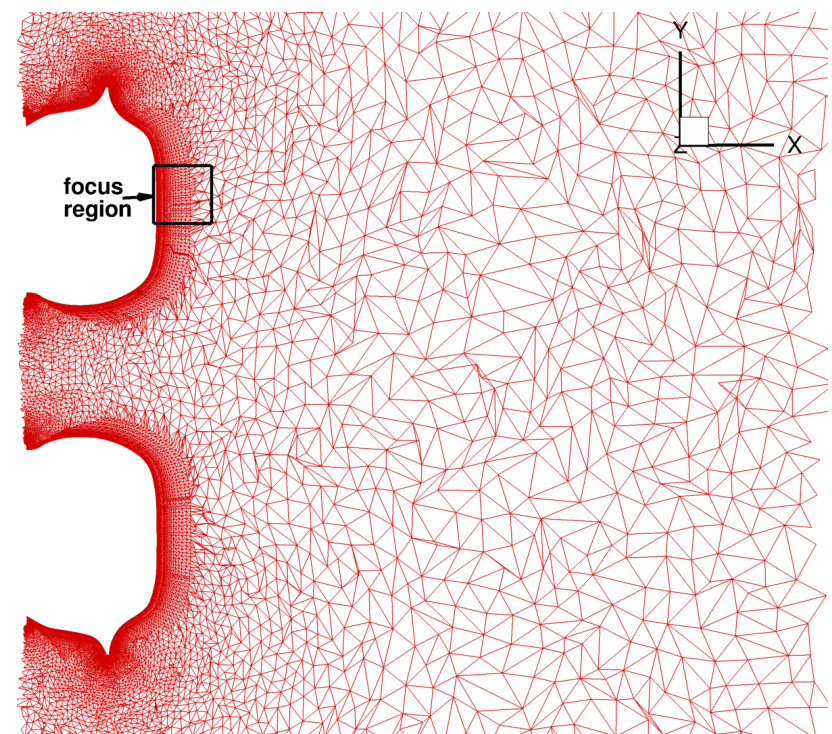

(a) Initial grid: 18M (adapt0)

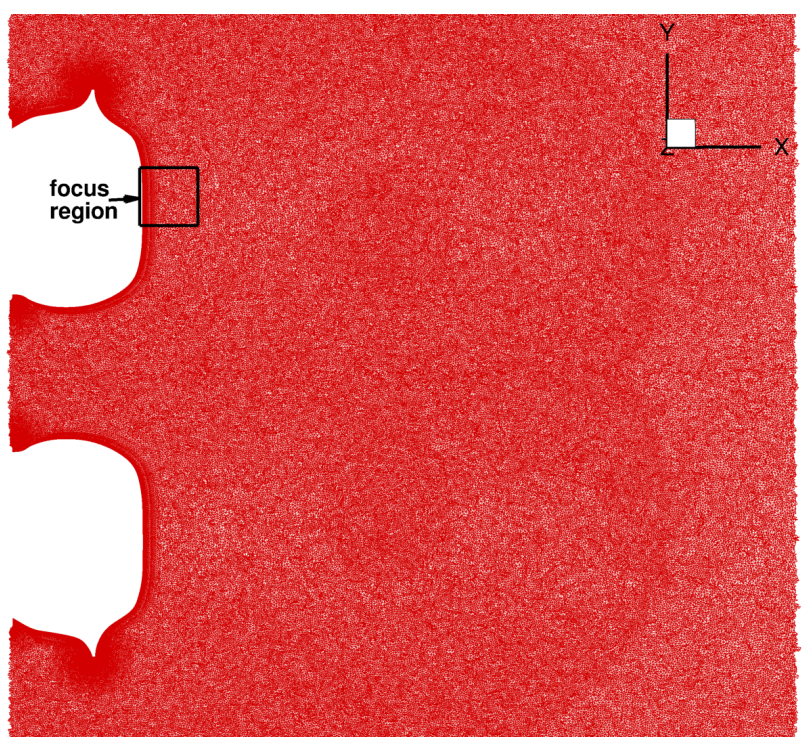

(b) Reference fine grid: $145 \mathrm{M}$

Figure 3. Comparison of mesh distribution at mid-section of tire wake, global view.

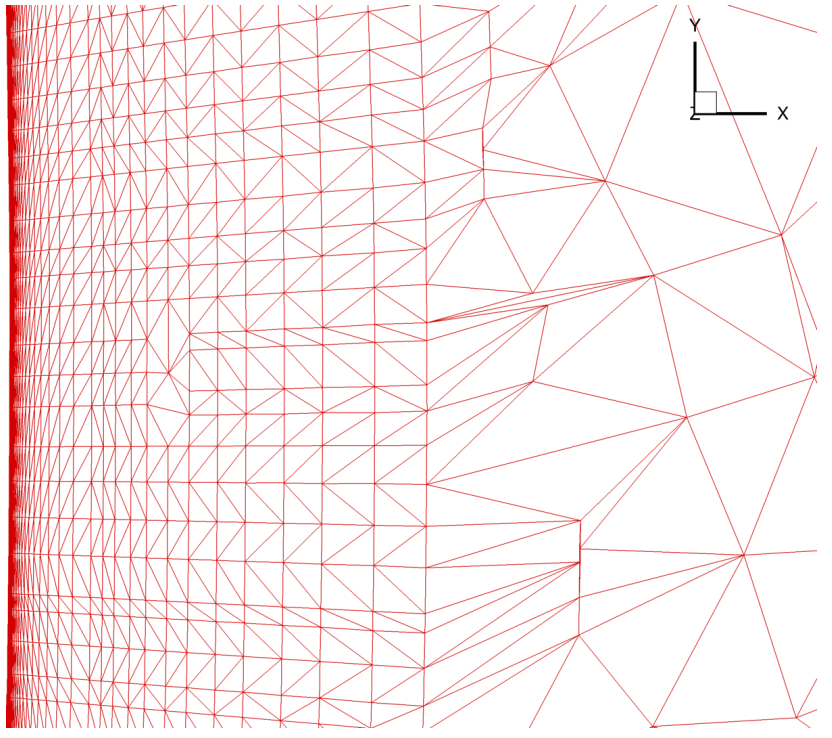

(a) Initial grid: 18M (adapt0)

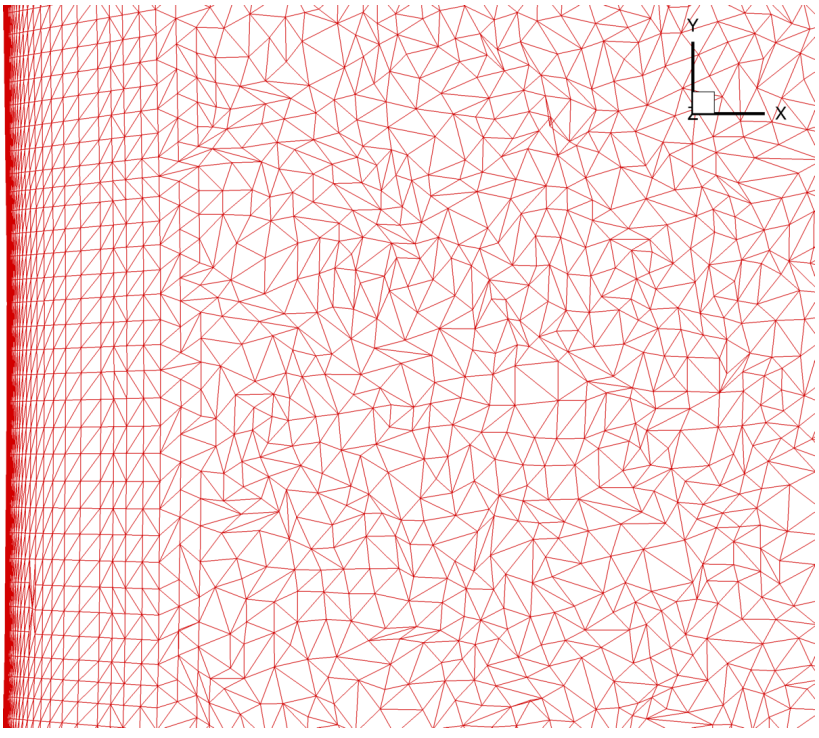

(b) Reference fine grid: $145 \mathrm{M}$

Figure 4. Comparison of mesh distribution at mid-section of tire wake, zoomed view of focus region identified in Fig. 3. 


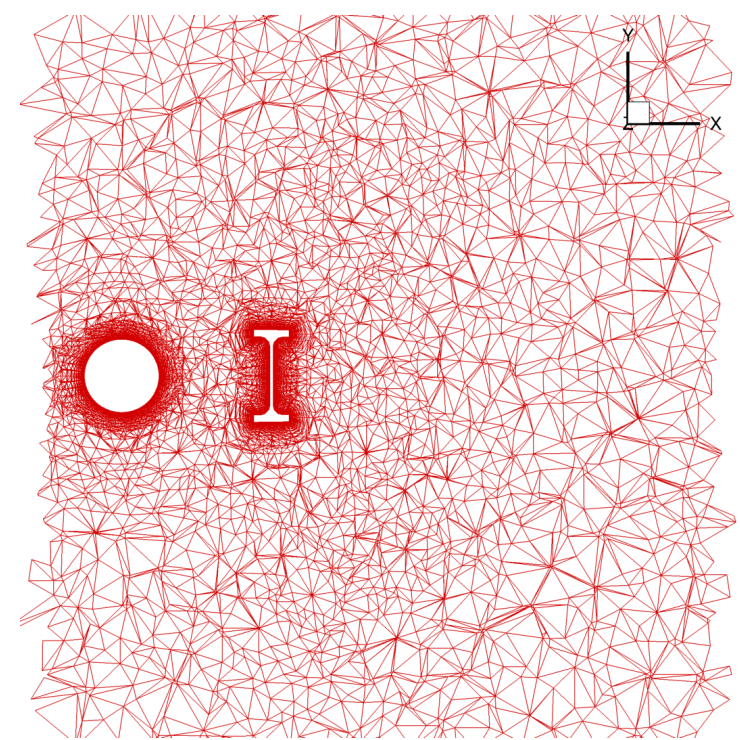

(a) Initial grid: 18M (adapt0)

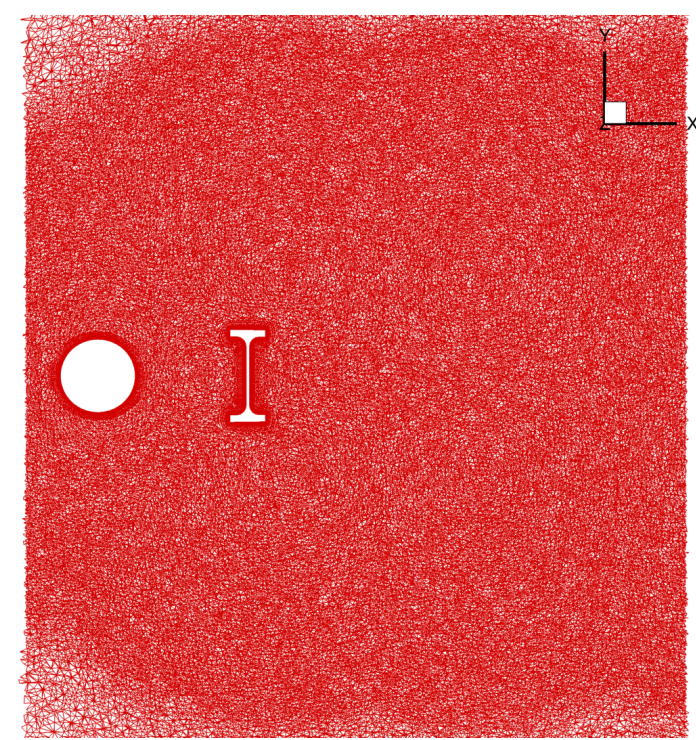

(b) Reference fine grid: $145 \mathrm{M}$

Figure 5. Comparison of initial grid with reference grid at torque-arm cut.

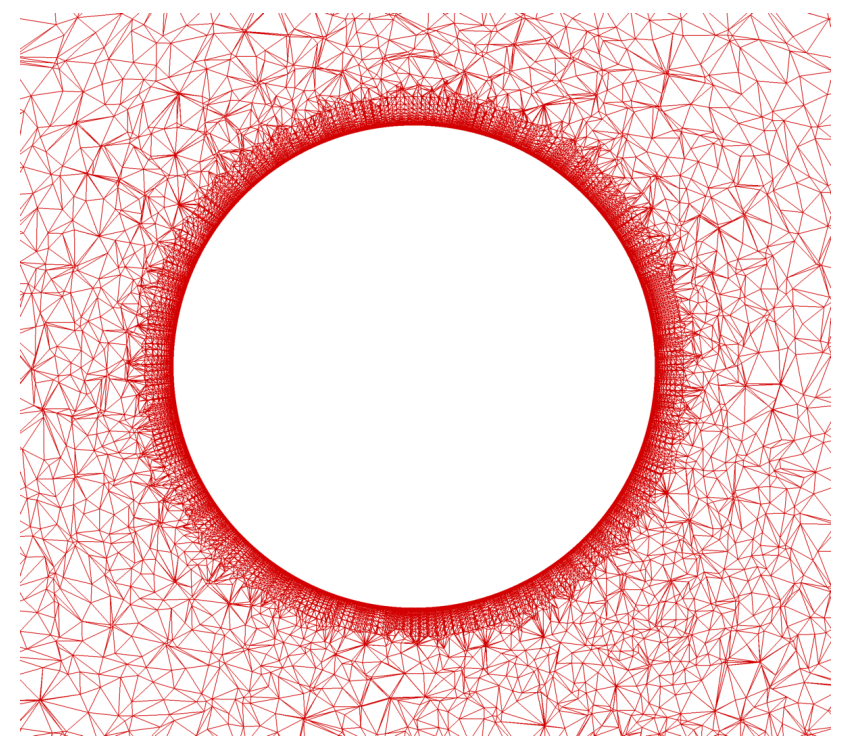

(a) Initial grid: 18M (adapt0)

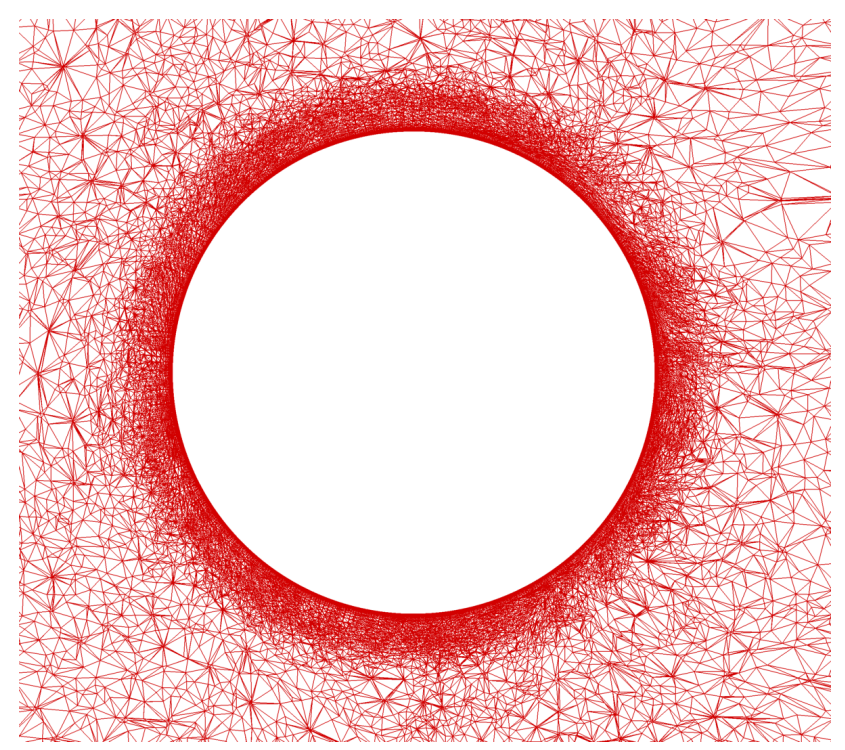

(b) 24M grid obtained with steady-state adjoint

Figure 6. Comparison of initial grid with steady adjoint based adapted grid at mid-section of tire. 


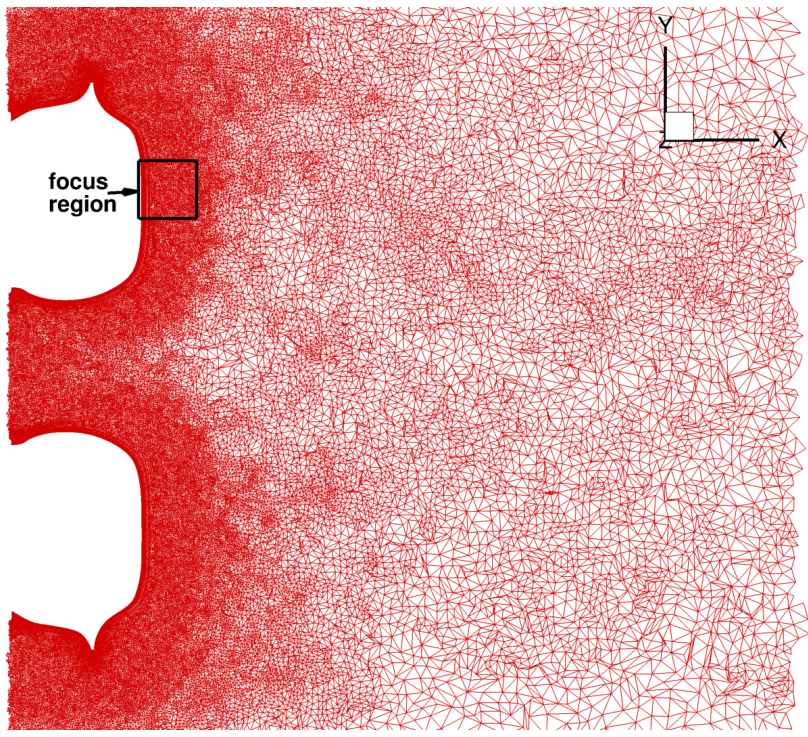

(a) Medium adapted grid: 40M (adapt1)

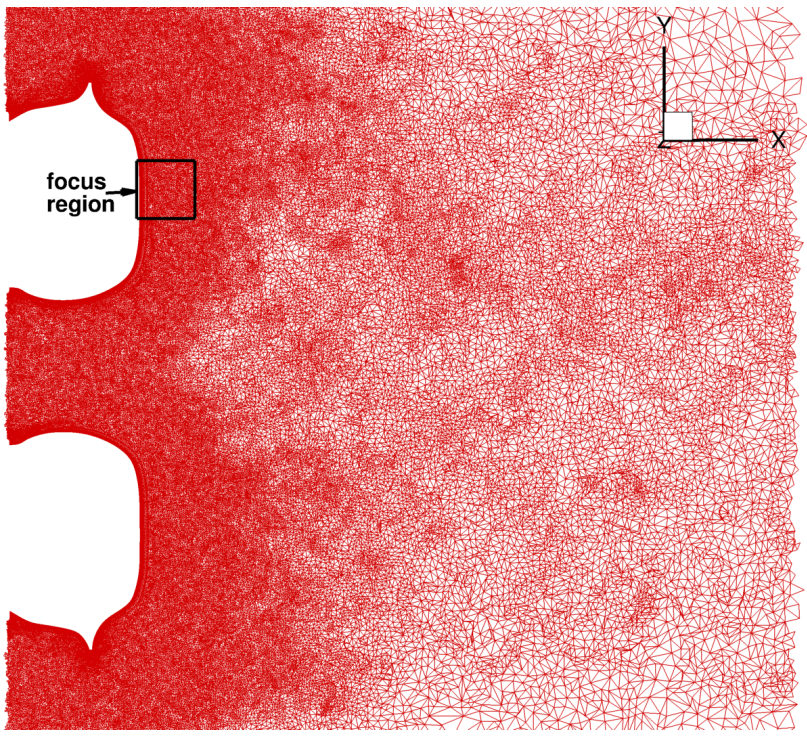

(b) Fine adapted grid: 58M (adapt2)

Figure 7. Comparison of mesh distribution at mid-section of tire wake for adapted grids, global view.

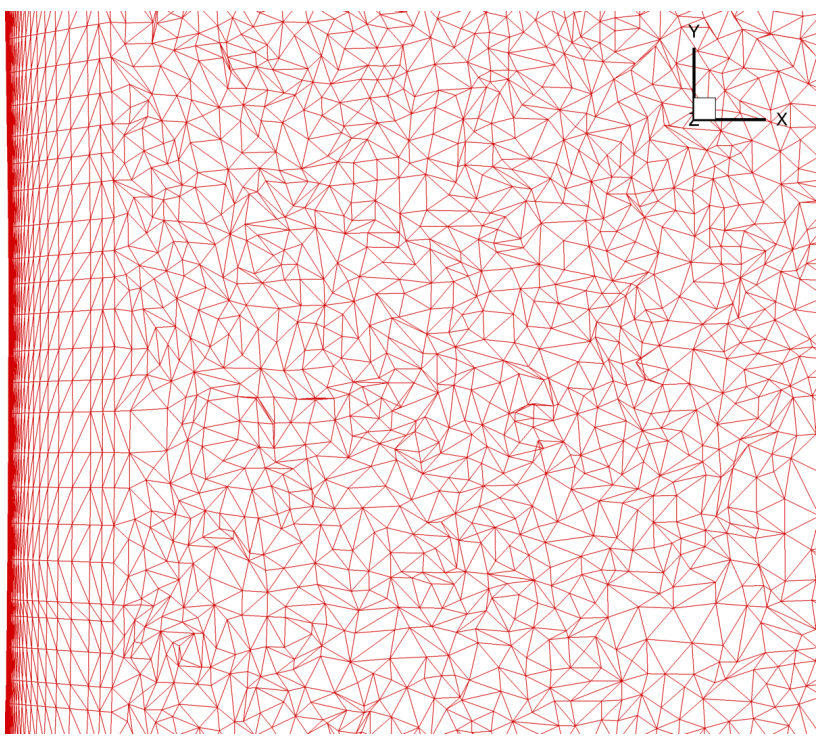

(a) Medium adapted grid: 40M (adapt1)

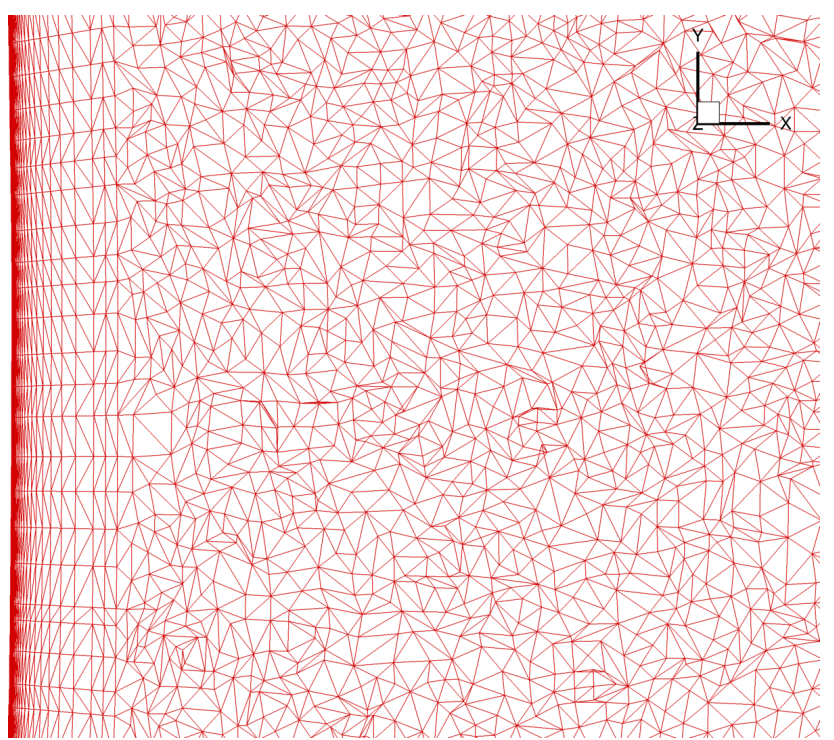

(b) Fine adapted grid: 58M (adapt2)

Figure 8. Comparison of mesh distribution at mid-section of tire wake for adapted grids, zoomed view of focus region identified in Fig. 7. 


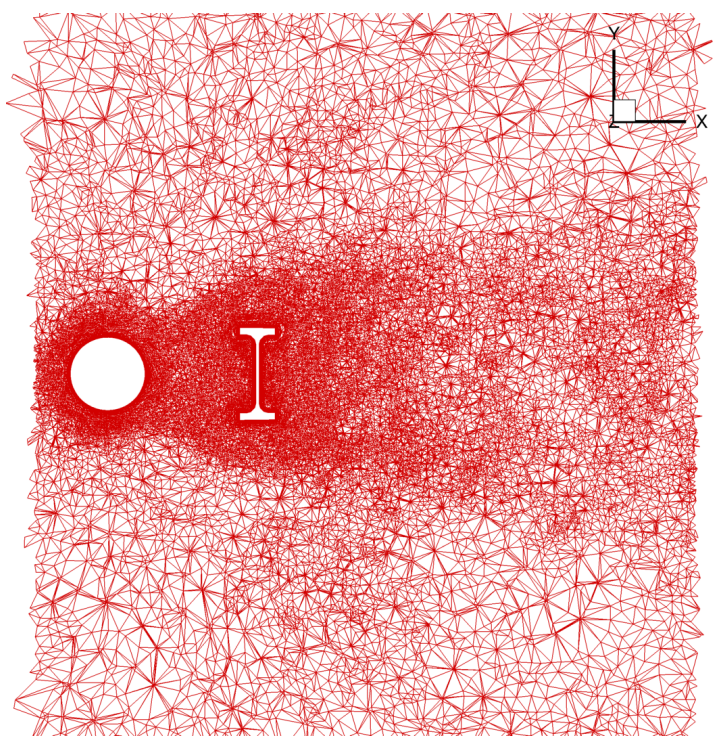

(a) Medium adapted grid: 40M (adapt1)

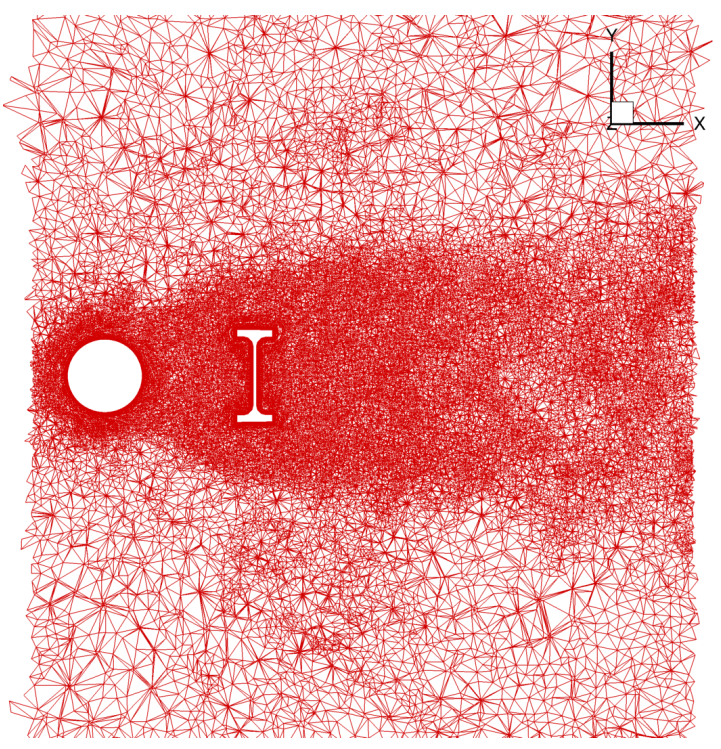

(b) Fine adapted grid: 58M (adapt2)

Figure 9. Comparison of adapted grids at torque-arm cut. 


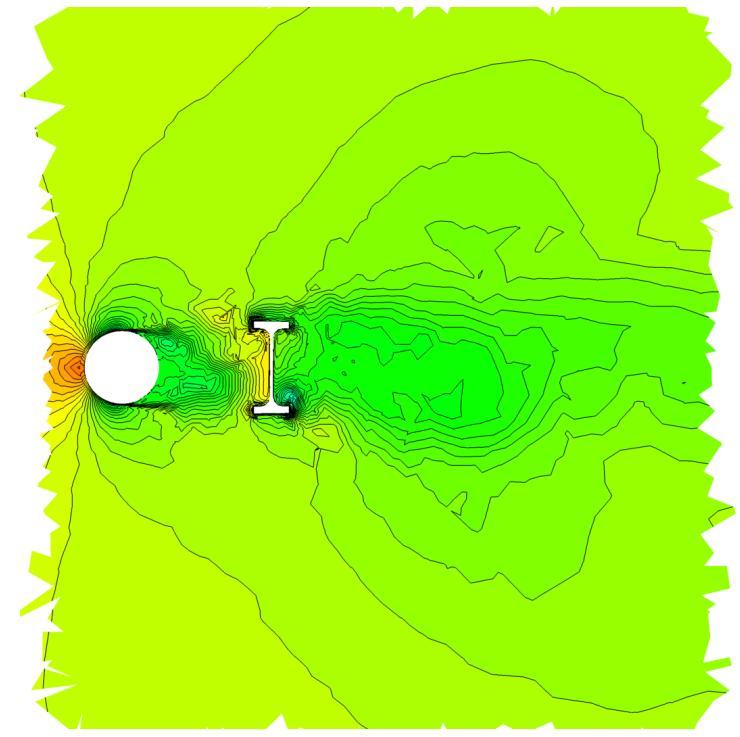

(a) Initial grid: 18M (adapt0)

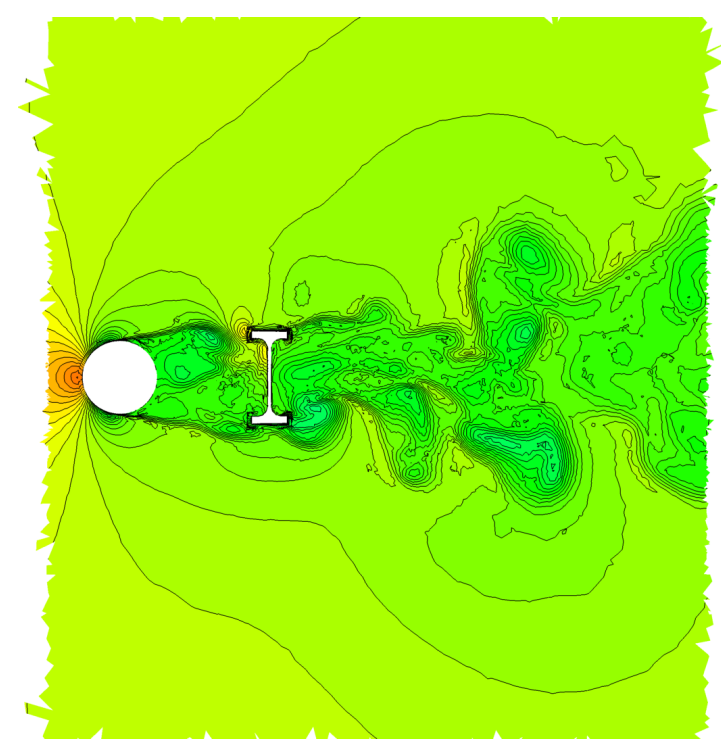

(c) Fine adapted grid: 58M (adapt2)

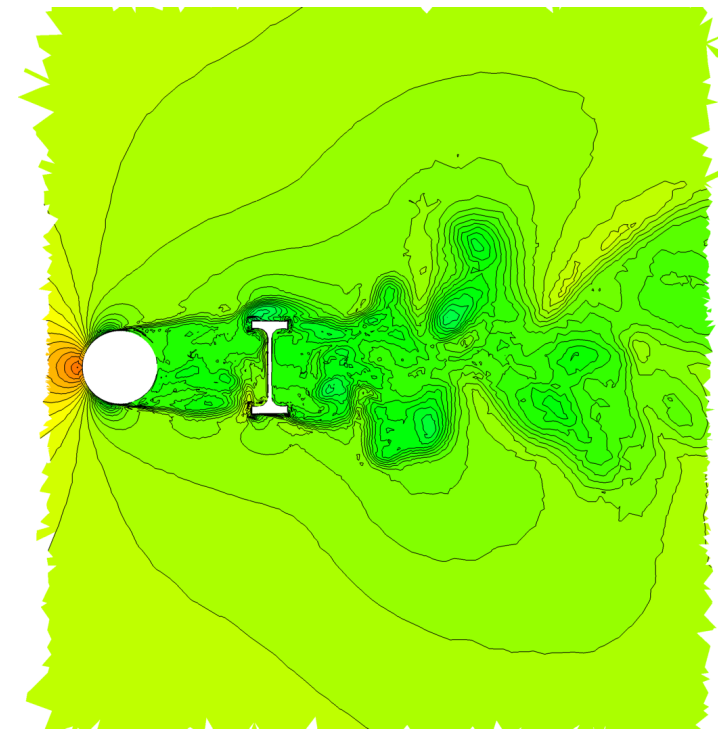

(b) Medium adapted grid: 40M (adapt1)

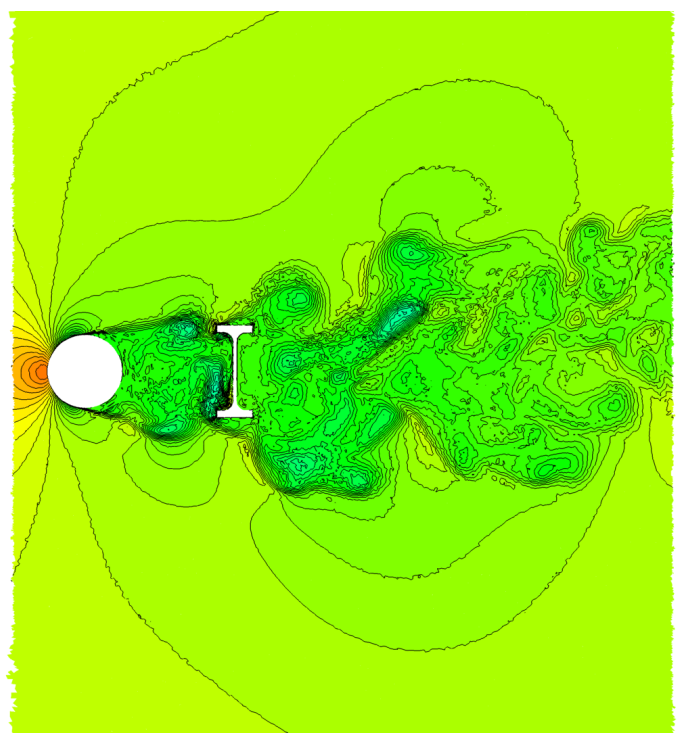

(d) Reference fine grid: $145 \mathrm{M}$

Figure 10. Comparison of instantaneous density contours at torque-arm cut. 


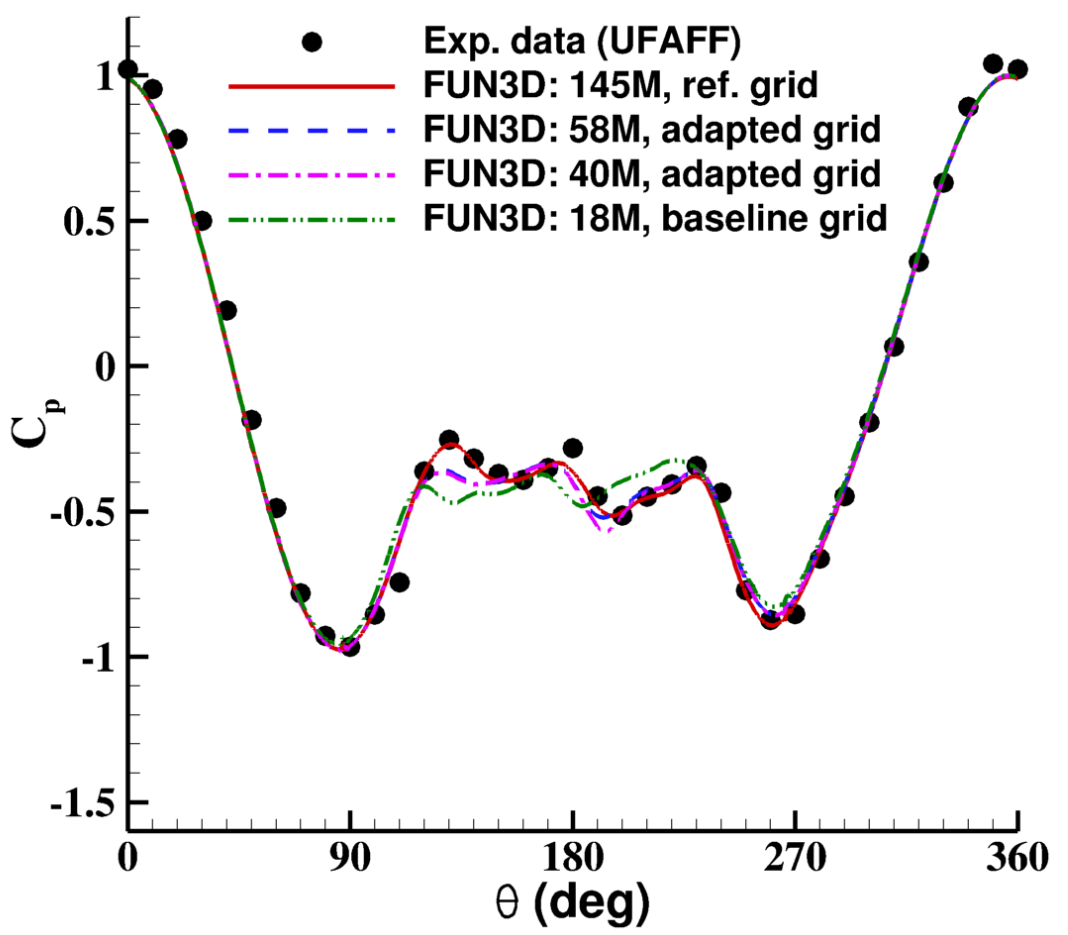

Figure 11. Surface pressure distributions on port wheel.

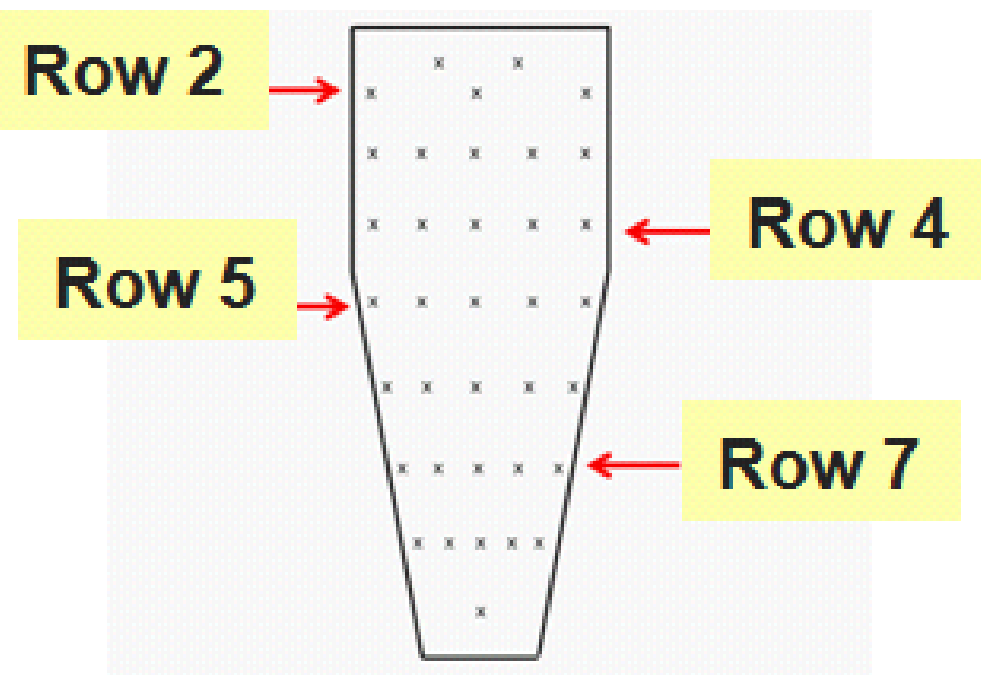

Figure 12. Nomenclature for pressure port rows on cavity door. 


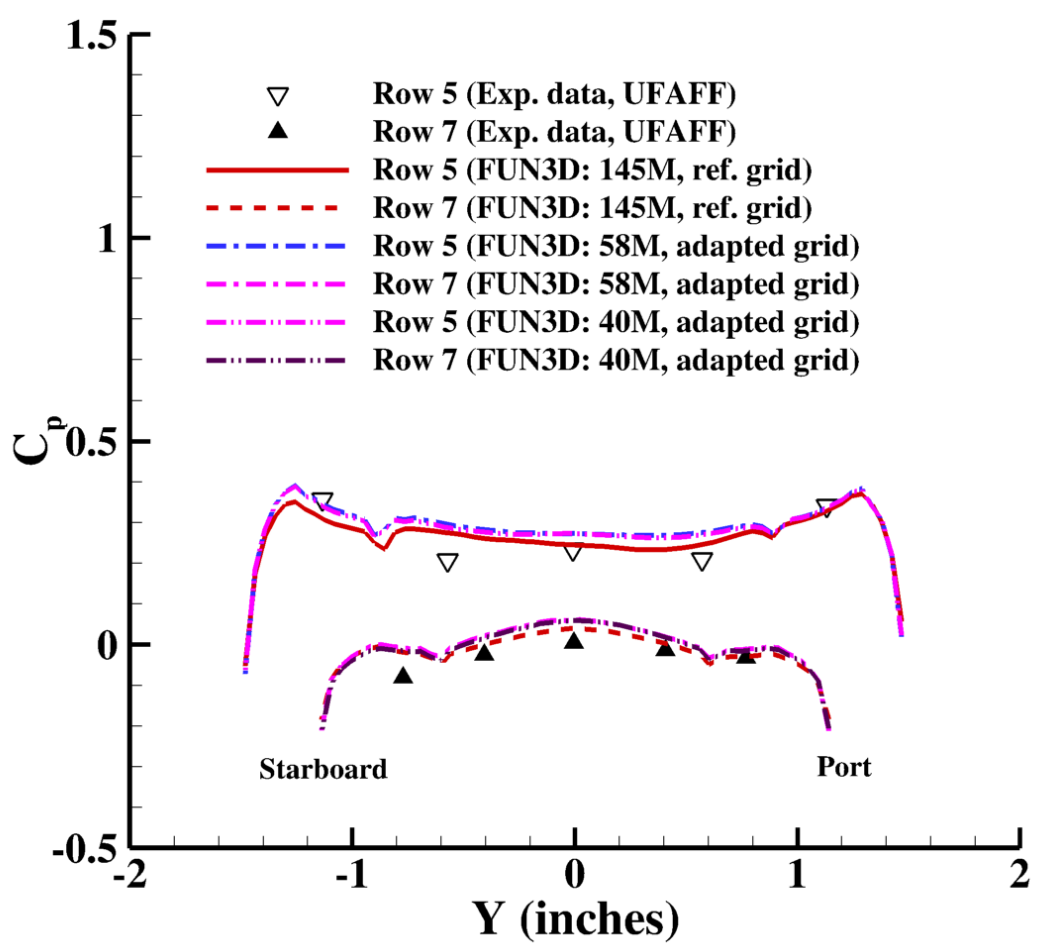

Figure 13. Surface pressure distributions on cavity door.

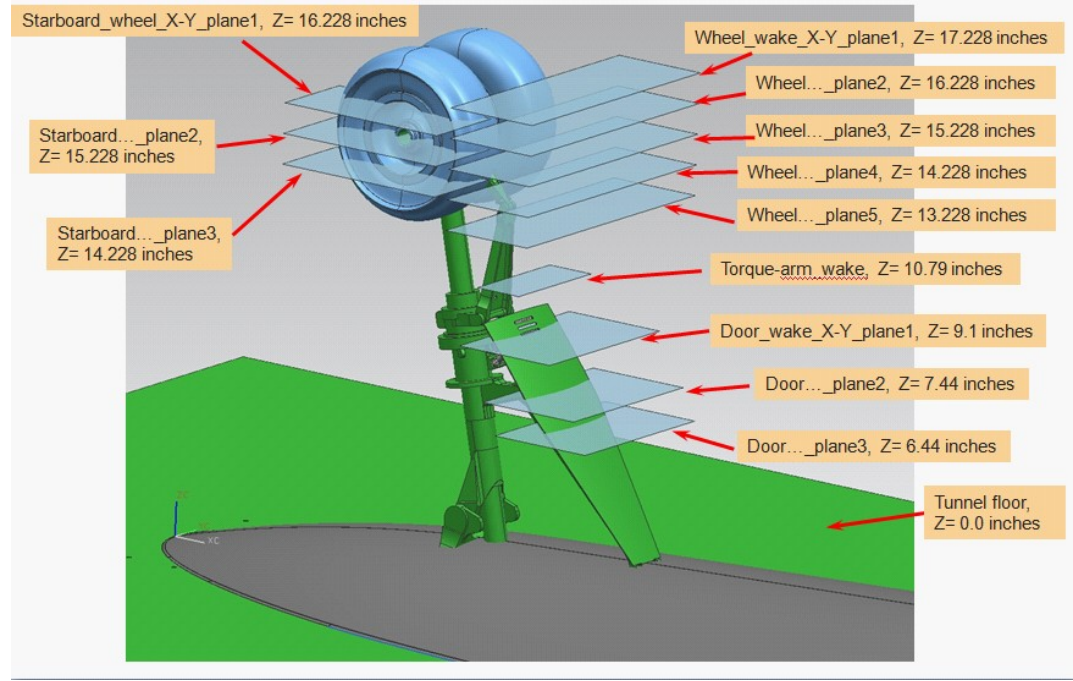

Figure 14. PIV measurement planes for BART experimental set up. 


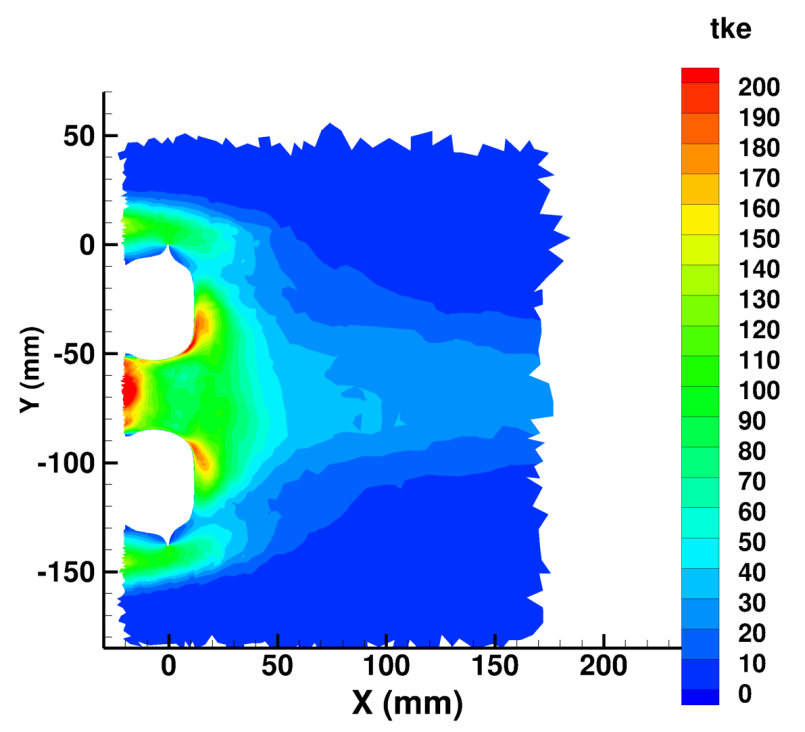

(a) FUN3D simulation, 18M

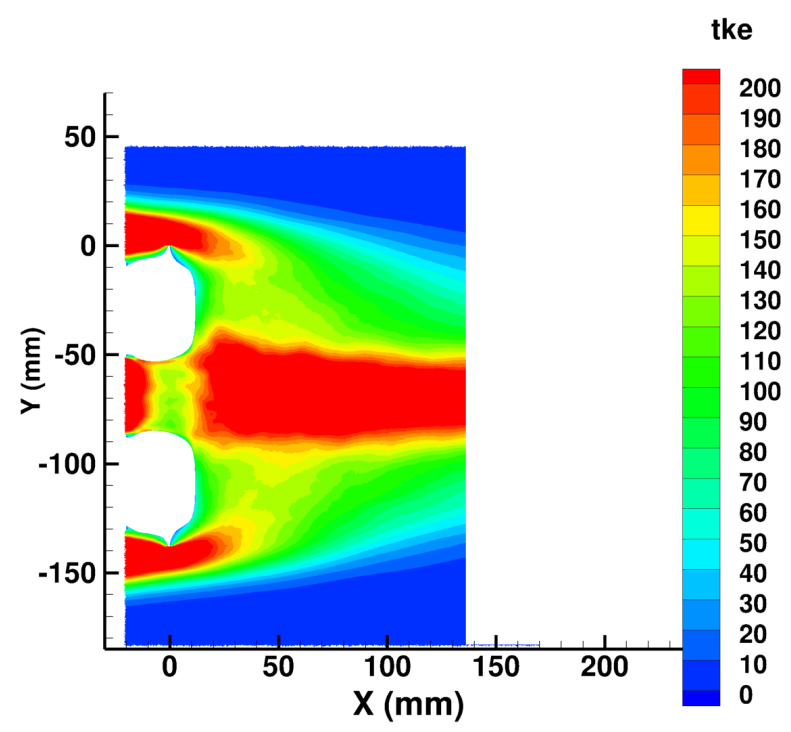

(c) FUN3D simulation, 145M

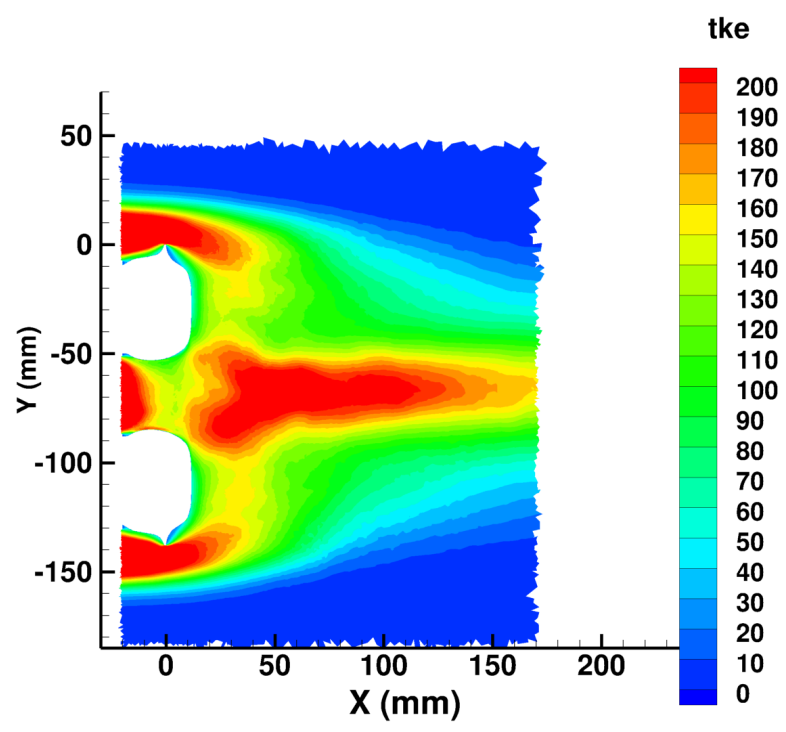

(b) FUN3D simulation, 58M

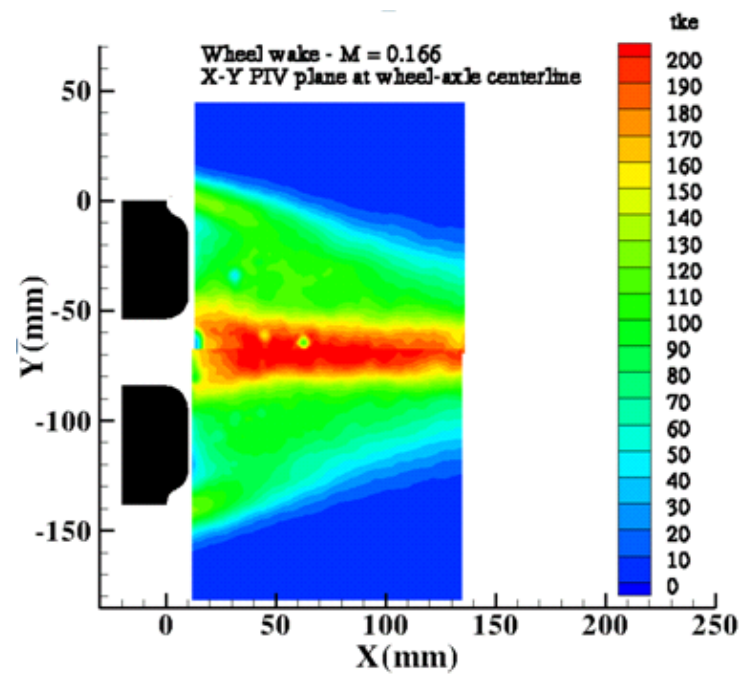

(d) Exp. PIV data

Figure 15. 2-D Turbulence kinetic energy contours, mid-wheel plane3 identified in Fig. 14. 


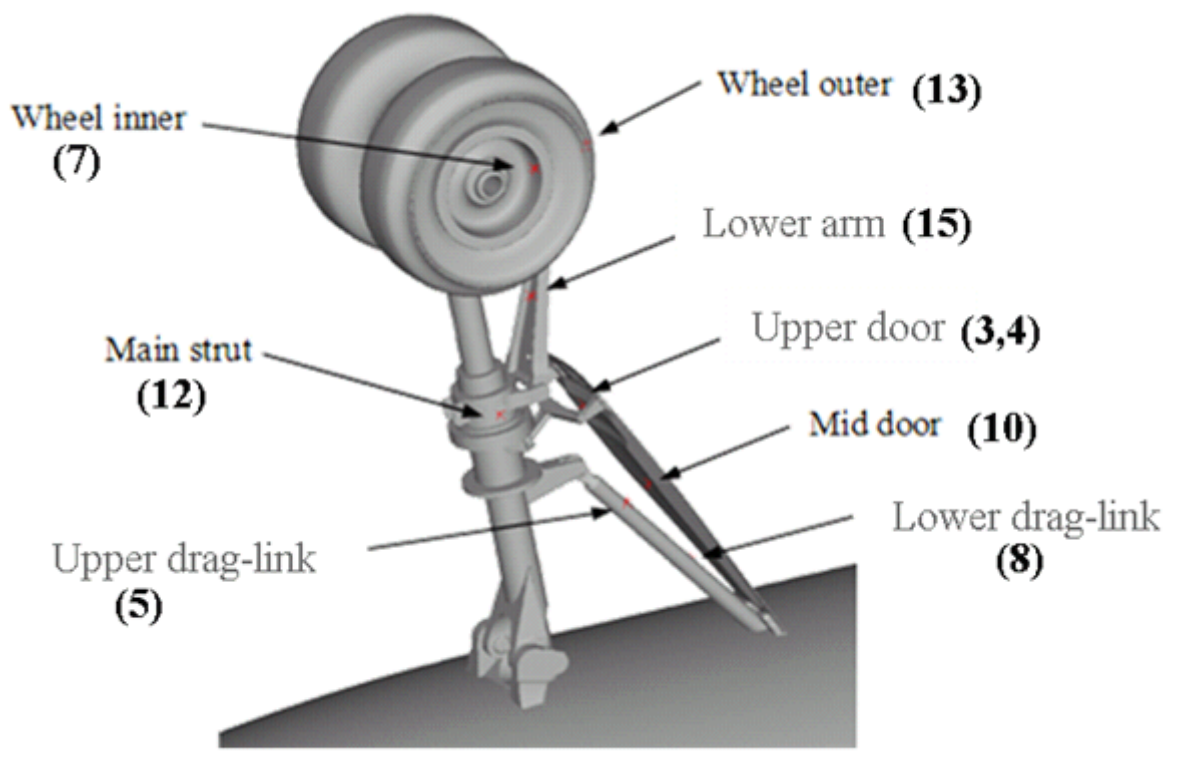

Figure 16. Surface mounted Kulite ${ }^{\circledR}$ sensor locations.
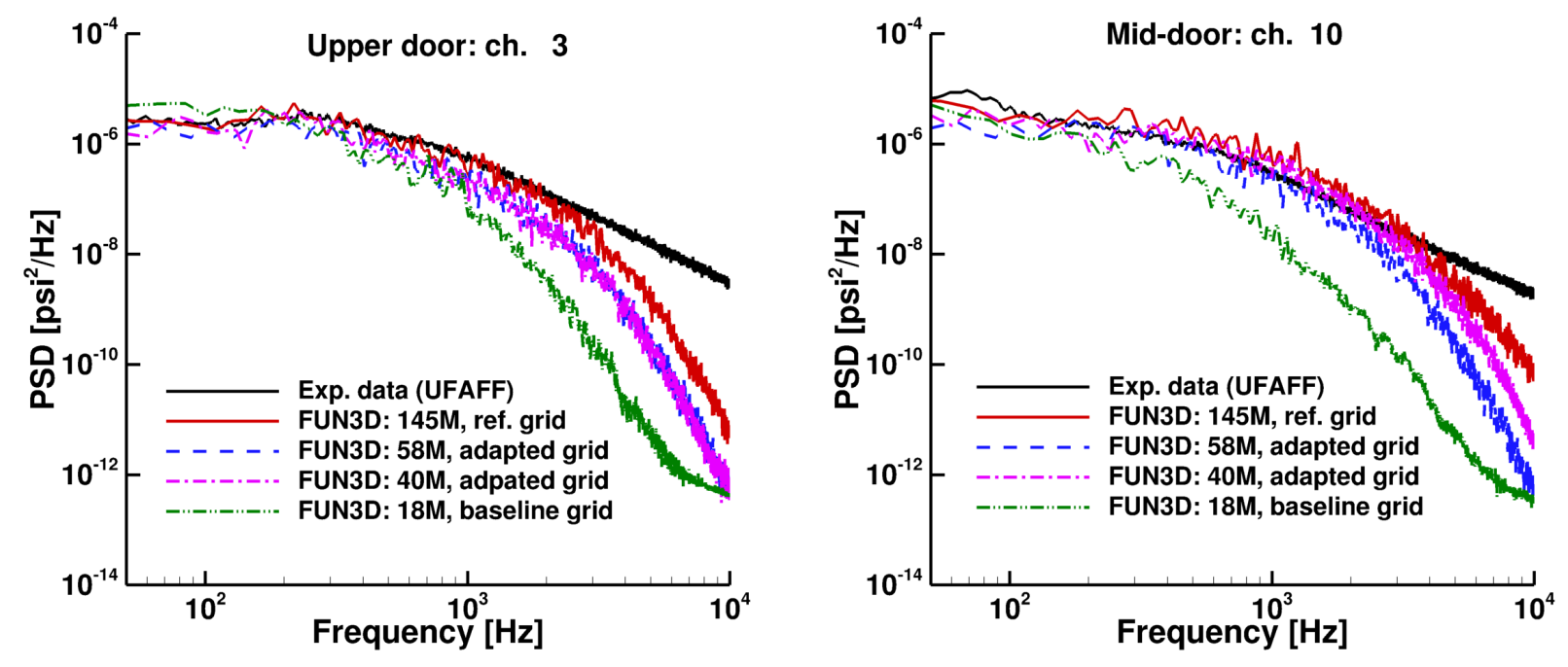

Figure 17. PSD comparisons for Kulite ${ }^{\circledR}$ sensors 3 and 10. 

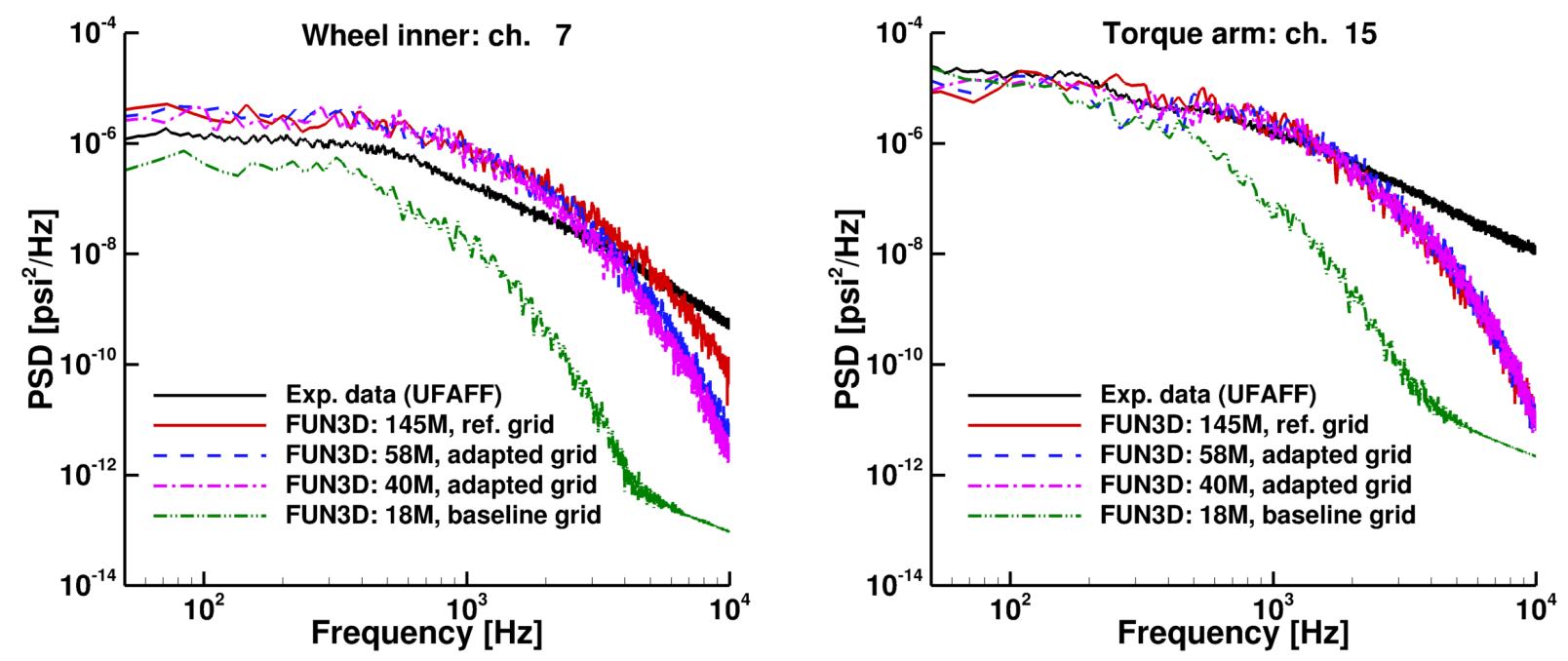

Figure 18. PSD comparisons for Kulite ${ }^{\circledR}$ sensors 7 and 15.

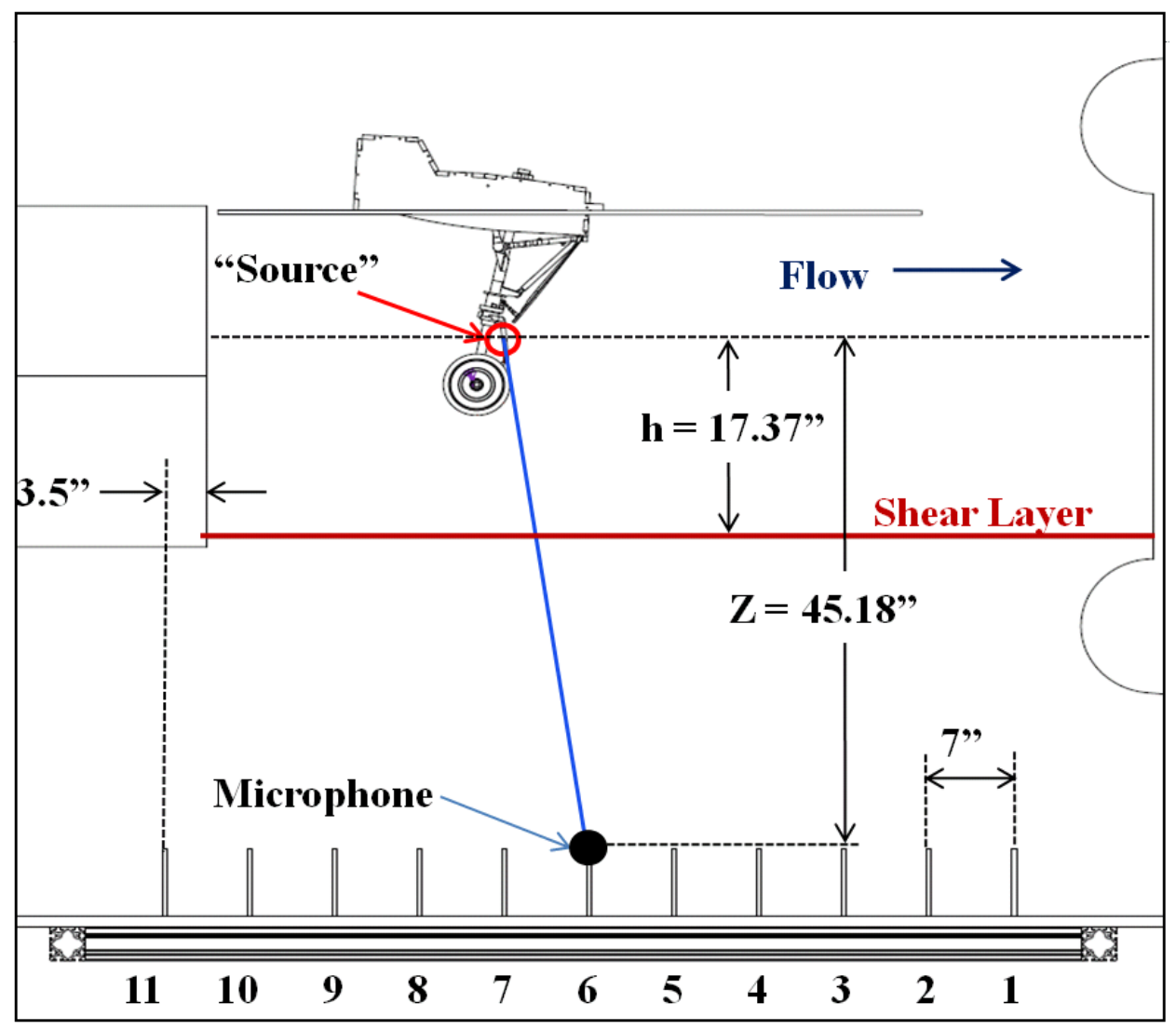

Figure 19. Linear microphone array arrangement in UFAFF. 


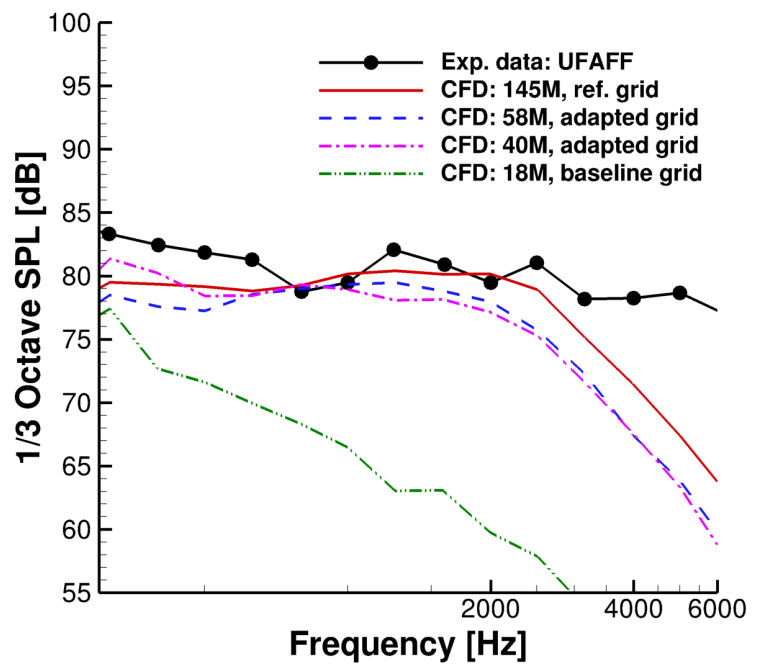

(a) Microphone 4

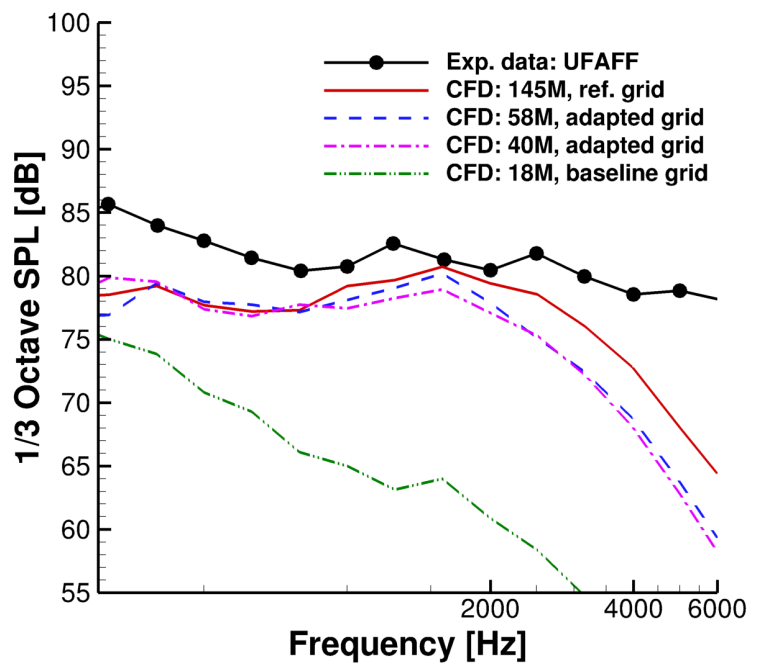

(b) Microphone 7

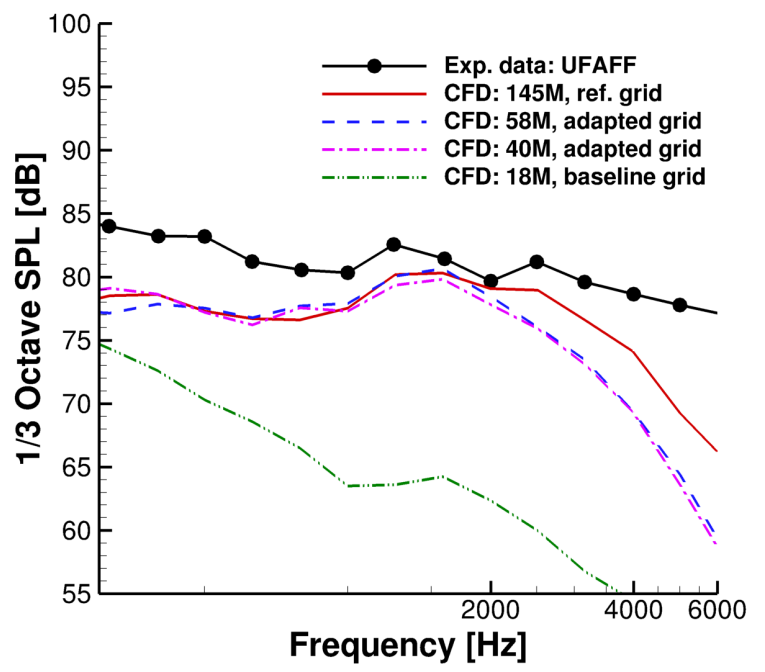

(c) Microphone 9

Figure 20. Sound pressure level comparisons at flyover microphones using solid surface perturbations in the FW-H Solver. 


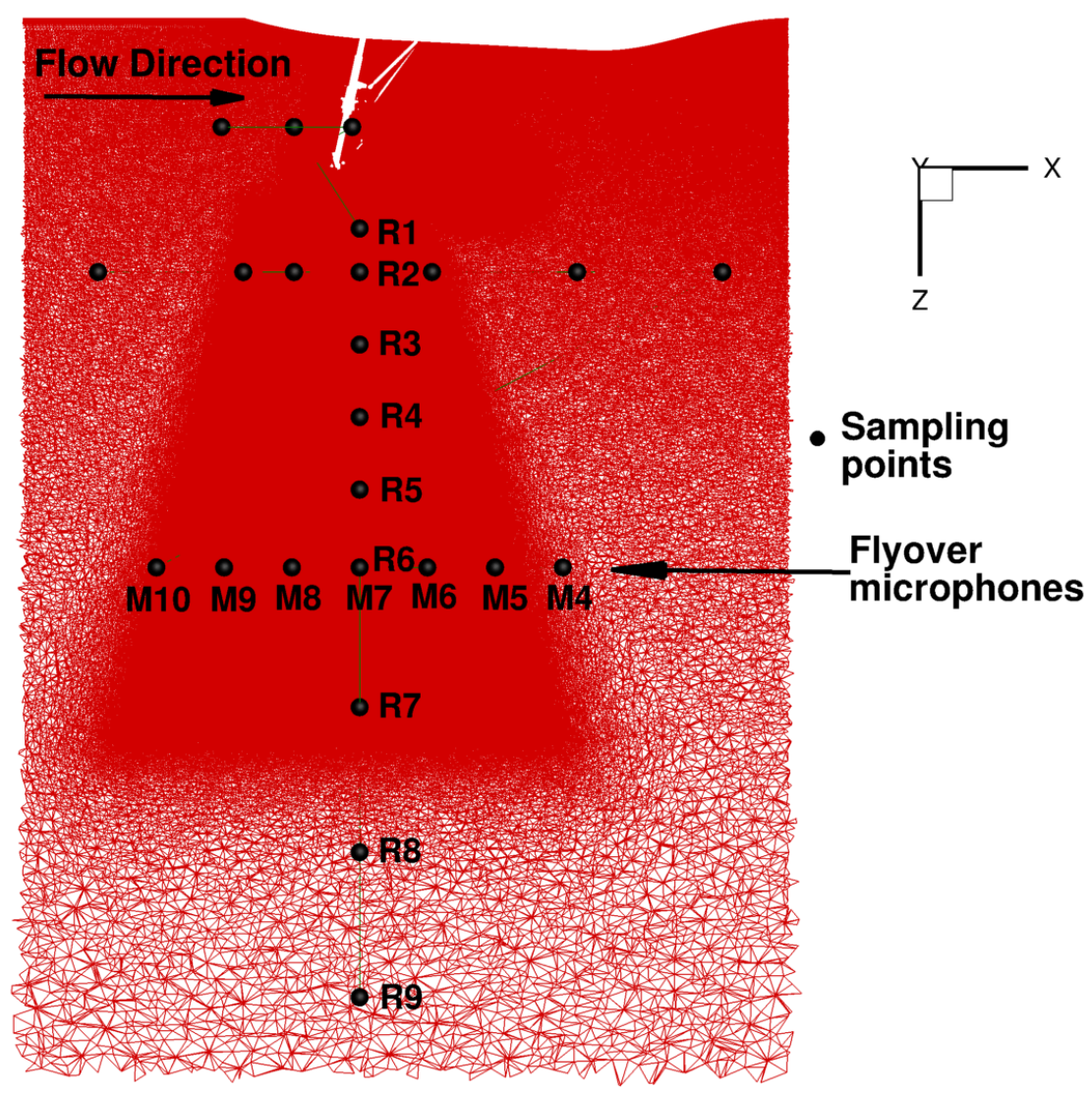

Figure 21. Sampling point locations for unsteady data collection, $y=0$ plane. 